Advances in Physics

Vol. 00, No. 00, January-February 2005, 1-54

\title{
New Trends in Density Matrix Renormalization
}

\author{
KAREN A. HALLBERG \\ 'Instituto Balseiro and Centro Atómico Bariloche, Comisión Nacional de Energía \\ Atómica, 8400 Bariloche, Argentina
}

(April 2006)

(1)

The Density Matrix Renormalization Group (DMRG) has become a powerful numerical method that _can be applied to low-dimensional strongly correlated fermionic and bosonic systems. It allows for $\rightleftarrows$ a very precise calculation of static, dynamic and thermodynamic properties. Its field of applicabilaty has now extended beyond Condensed Matter, and is successfully used in Quantum Chemistry, Statistical Mechanics, Quantum Information Theory, Nuclear and High Energy Physics as well. In this article, we briefly review the main aspects of the method and present some of the most relevant applications so as to give an overview on the scope and possibilities of DMRG. We focus on T the most important extensions of the method such as the calculation of dynamical properties, the application to classical systems, finite temperature simulations, phonons and disorder, field theory, time-dependent properties and the ab initio calculation of electronic states in molecules. The recent quantum information interpretation, the development of highly accurate time-dependent algorithms and the possibility of using the DMRG as the impurity-solver of the Dynamical Mean Field Method (DMFT) give new insights into its present and potential uses. We review the numerous very recent applications of these techniques where the DMRG has shown to be one of the most reliable and vèrsatile methods in modern computational physics.

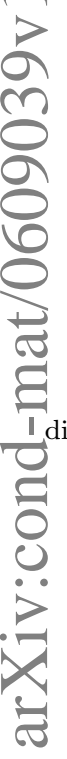

Keywords: density matrix renormalization; numerical methods; strongly correlated electrons; lowdimensional systems, quantum information 


\section{Contents}

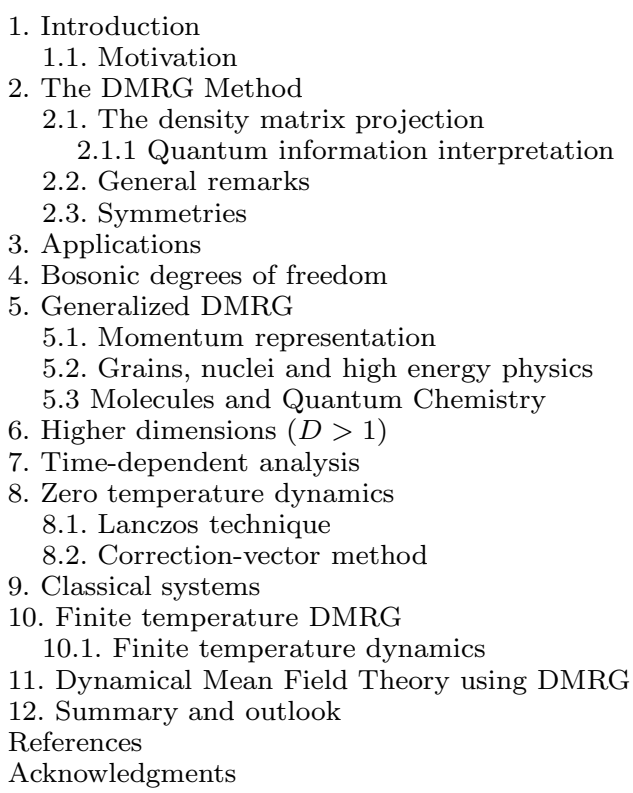

\section{Introduction}

The Density Matrix Renormalization Group (DMRG) was developed by S. White in 1992 [1] and since then it has proved to be a very powerful method for low dimensional interacting systems. Its remarkable accuracy can be seen for example in the spin-1 Heisenberg chain: for a system of hundreds of sites a precision of $10^{-10}$ for the ground state energy can be achieved. Since then it has been applied to a great variety of systems and problems including, among others, spin chains and ladders, fermionic and bosonic systems, disordered models, impurities, molecules, nanoscopic systems and 2D electrons in high magnetic fields. It has also been improved substantially in several directions like two dimensional (2D) classical systems, stochastic models, inclusion of phonons, quantum chemistry, field theory, finite temperature and the calculation of dynamical and time-dependent properties. Some calculations have also been performed in 2D quantum systems. Most of these topics are treated in detail and in a pedagogical way in the book 2, where the reader can find an extensive overview on the foundations of DMRG and also in a comprehensive review 3]. Recent new developments of the DMRG like the implementation of very accurate methods for time-dependent problems, the quantum information and matrix-product perspective and the possibility to combine it with the Dynamical Mean Field Theory (DMFT), triggered a great activity using these 
techniques, as seen by the numerous recent papers published in the last year. In this article we will mainly focus on these new developments and applications and hope to give new insights into its potential uses. In order to achieve this and on behalf of coherence we will also briefly describe the method and its former extensions.

\section{$1.1 \quad$ Motivation}

When considering finite systems, the exponential growth of degrees of freedom to be considered imposes an important limitation in numerical calculations. Several methods have been introduced in order to reduce the size of the Hilbert space to be able to reach larger systems, such as Monte Carlo, renormalization group (RG) and DMRG. Each method considers a particular criterion for keeping the relevant information.

The DMRG was originally developed to overcome the problems that arise in interacting systems in $1 \mathrm{D}$ when standard RG procedures were applied. For example, consider a block B (a block is a collection of sites) where the Hamiltonian $H_{B}$ and end-operators are defined. These traditional methods consist in putting together two or more blocks (e.g. B-B', which we will call the superblock), connected using end-operators, in a basis that is a direct product of the basis of each block, forming $H_{B B^{\prime}}$. This Hamiltonian is then diagonalized, the superblock is replaced by a new effective block $B_{\text {new }}$ formed by a certain number $m$ of lowest-lying eigenstates of $H_{B B^{\prime}}$ and the iteration is continued (see Ref. 4]). Although it has been used successfully in certain cases, this procedure, or similar versions of it, has been applied to several interacting systems with poor performance. For example, it has been applied to the 1D Hubbard model keeping $m \simeq 1000$ states and for 16 sites, an error of $5-10 \%$ was obtained [5]. Other results [6] were also discouraging. A better performance was obtained [7] by adding a single site at a time rather than doubling the block size. However, there is one case where a similar version of this method applies very well: the impurity problem represented by the Kondo and Anderson models. Wilson 8] mapped the one-impurity problem onto a one-dimensional lattice with exponentially decreasing hoppings. The difference with the method explained above is that in this case, one site (equivalent to an "onion shell") is added at each step and, due to the exponential decrease of the hopping, very accurate results can be obtained. A very recent work on renormalization group transformations on quantum states based on matrix product states was performed in Ref. [9].

Returning to the problem of putting several blocks together, the main source of error comes from the election of eigenstates of $H_{B B^{\prime}}$ as representative states of a superblock. Since $H_{B B^{\prime}}$ has no connection to the rest of the lattice, its eigenstates may have unwanted features (like nodes) at the ends of the block 
and this can't be improved by increasing the number of states kept. Based on this consideration, Noack and White [10] tried including different boundary conditions and boundary strengths. This turned out to work well for single particle and Anderson localization problems but, however, it did not improve the results significantly for interacting systems. These considerations led to the idea of taking a larger superblock that includes the blocks $B B^{\prime}$, diagonalize the Hamiltonian in this large superblock and then somehow project the most favorable states onto $B B^{\prime}$. Then $B B^{\prime}$ is replaced by $B_{\text {new }}$. In this way, awkward features in the boundary would vanish and a better representation of the states in the infinite system would be achieved. White [1,4] appealed to Feynman's formulation of the density matrix as the best description of a part of a quantum mechanical system and, thus, as the optimal way of projecting the most relevant states onto a subsystem. In Ref. 11], Gaite shows that, by using an angular quantization construction of the density matrix, the DMRG is an algorithm that keeps states with a higher weight near the boundaries in a systematic way. This is a consequence of the fact that, near the boundaries, there is a concentration of quantum states, in a similar manner as in the physics of black holes.

In the following Section we will describe the standard method; in Sect. 3 we will mention some of the most important applications, describing in Sect. 4 the treatment of bosonic degrees of freedom. In Sec. 5 we review the most relevant extensions of the method like the momentum and energy levels representation and the application to Quantum Chemistry. The improvement to treat systems in dimensions higher than 1 is mentioned in Sec. 6, while Sect. 7 will be devoted to the latest and very promising developments to calculate non-equilibrium and time-dependent properties. In Sect. 8 we deal with the calculation of dynamical properties at zero temperature and in Sect. 9 we briefly describe how DMRG can be used to calculate physical properties of classical systems. In connection to this, finite temperature studies were possible within DMRG and this is explained in Section 10. In the following Sect. 11 we describe how the Dynamical Mean Field Theory (DMFT) can profit from the DMRG as an impurity-solver to obtain spectral properties of correlated systems, opening new posibilities for the calculation of more complicated and realistic systems. Finally, we summarize the achievements of the method and look upon new potential applications and developments.

\section{The DMRG Method}

The DMRG allows for a systematic truncation of the Hilbert space by keeping the most probable states describing a wave function (e.g. the ground state) instead of the lowest energy states usually kept in previous real space renor- 


\section{System Environment}

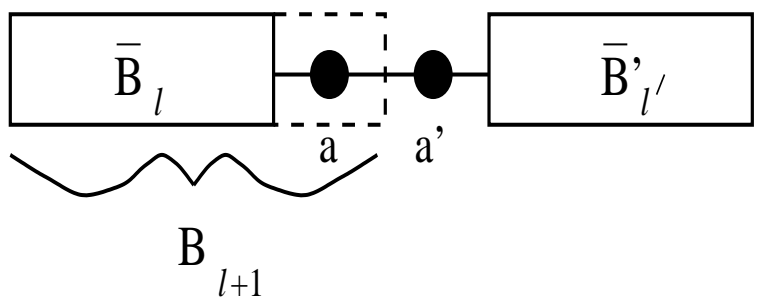

Figure 1. A scheme of the superblock (universe) configuration for the DMRG algorithm [4.

malization techniques.

The basic idea consists in starting with a small system (e.g with $N$ sites) and then gradually increase its size (to $N+2, N+4, \ldots$ ) until the desired length is reached. Let us call the collection of $N$ sites the universe and divide it into two parts: the system and the environment (see Fig. 1). The Hamiltonian is constructed in the universe and its ground state $\left|\psi_{0}\right\rangle$ is obtained. This is considered as the state of the universe and called the target state. It has components on the system and the environment. We want to obtain the most relevant states of the system, i.e., the states of the system that have largest weight in $\left|\psi_{0}\right\rangle$. To obtain this, the environment is considered as a statistical bath and the density matrix [12] is used to obtain the desired information on the system. So instead of keeping eigenstates of the Hamiltonian in the block (system), we keep eigenstates of the density matrix. We will be more explicit below.

A very easy and pedagogical way of understanding the basic functioning of DMRG is applying it to the calculation of simple quantum problems like one particle in a tight-binding chain [13,14]. In these examples, a discretized version of the Schrödinger equation is used and extremely accurate results are obtained for the quantum harmonic oscillator, the anharmonic oscillator and the double-well potential.

Let's define block $[\mathbf{B}]$ as a finite chain with $l$ sites having an associated Hilbert space with $M$ total amount of states (or $m$ states after a reduction is performed) where operators are defined (in particular the Hamiltonian in this finite chain, $H_{B}$, and the operators at the ends of the block, useful for linking it to other chains or added sites). Except for the first iteration, the basis in this block isn't explicitly known due to previous basis rotations and reductions. The operators in this basis are matrices and the basis states are characterized by quantum numbers (like $S^{z}$, charge or number of particles, etc). We also define an added block or site as [a] having $n$ states. A general 
iteration of the method is described below:

i) Define the Hamiltonian $H_{B B^{\prime}}$ for the superblock (the universe) formed by putting together two blocks $[\mathbf{B}]$ and $\left[\mathbf{B}^{\prime}\right]$ and two added sites $[\mathbf{a}]$ and $\left[\mathbf{a}^{\prime}\right]$ in this way: [B a a' $\mathbf{B}$ ' ], where the primes indicate additional blocks. The primed blocks usually have the same structure as the non-primed ones, but this can vary (see the finite-system algorithm below). The block [B'] has $M^{\prime}$ or $m^{\prime}$ states for the full and reduced spaces, respectively. In general, blocks $[\mathbf{B}]$ and $\left[\mathbf{B}^{\prime}\right]$ come from the previous iteration. The total Hilbert space of this superblock is the direct product of the individual spaces corresponding to each block and the added sites. In practice a quantum number of the superblock can be fixed (in a spin chain for example one can look at the total $S^{z}=0$ subspace), so the total number of states in the superblock is much smaller than $(m n)^{2}$. In some cases, as the quantum number of the superblock consists of the sum of the quantum numbers of the individual blocks, each block must contain several subspaces (several values of $S^{z}$ for example). Here periodic boundary conditions can be attached to the ends and a different block layout should be considered (e.g. [B a B' a' ]) to avoid connecting blocks $[\mathbf{B}]$ and [B'] which takes longer to converge. The boundary conditions are between [a'] and $[\mathbf{B}]$. For closed chains the performance is poorer than for open boundary conditions 4,15] (see Fig. 2)

ii) Diagonalize the Hamiltonian $H_{B B^{\prime}}$ to obtain the ground state $\left|\psi_{0}\right\rangle$ (target state) using Lanczos 16 or Davidson 17 algorithms. Other states could also be kept, such as the first excited ones: they are all called target states. A faster convergence of Lanczos or Davidson algorithm is achieved by choosing a good trial vector 18, 19.

iii) Construct the density matrix:

$$
\rho_{i i^{\prime}}=\sum_{j} \psi_{0, i j} \psi_{0, i^{\prime} j}
$$

on block [B a], where $\psi_{0, i j}=\left\langle i \otimes j \mid \psi_{0}\right\rangle$, the states $|i\rangle$ belonging to the Hilbert space of the block [ $\mathbf{B}$ a] and the states $|j\rangle$ to the block [B' $\left.\mathbf{a}^{\prime}\right]$. The density matrix considers the part $[\mathbf{B} \mathbf{a}]$ as a system and [B' $\left.\mathbf{a}^{\prime}\right]$, as a statistical bath. The eigenstates of $\rho$ with the highest eigenvalues correspond to the most probable states (or equivalently the states with highest weight) of block $[\mathbf{B} \mathbf{a}]$ in the ground state of the whole superblock. These states are kept up to a certain cutoff, keeping a total of $m$ states per block. The density matrix eigenvalues, $\omega_{\alpha}$, sum up to unity and the truncation error, defined as the sum of the density matrix eigenvalues corresponding to discarded eigenvectors, $\sum_{\alpha=m+1}^{m_{\max }} \omega_{\alpha}=1-\operatorname{sum}_{\alpha=1}^{m} \omega_{\alpha}$, gives a qualitative indication of the accuracy of the calculation $\left(m_{\max }\right.$ corresponds to the size of the full space of block $\left.[\mathbf{B} \mathbf{a}]\right)$.

iv) With these $m$ states a rectangular matrix $O$ is formed and it is used to 


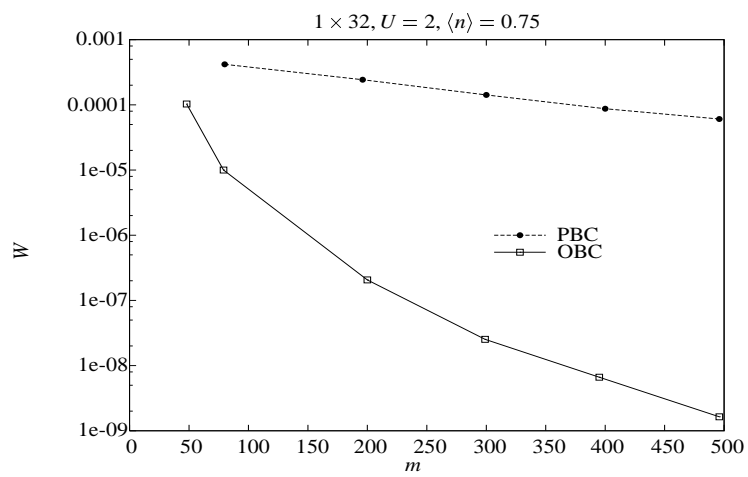

Figure 2. Truncation error for the finite-system DMRG as a function of the number of states kept $m$ (reprinted from [13, with permission).

change basis and reduce all operators defined in $[\mathbf{B} \mathbf{a}]$. This block $[\mathbf{B} \mathbf{a}]$ is then renamed as block $\left[\mathbf{B}_{\text {new }}\right]$ or simply $[\mathbf{B}]$ (for example, the Hamiltonian in block $[\mathbf{B}$ a $], H_{B a}$, is transformed into $H_{B}$ as $\left.H_{B}=O^{\dagger} H_{B a} O\right)$.

v) A new block $[\mathbf{a}]$ is added (one site in our case) and the new superblock [B $\mathbf{a}$ a' $\left.\mathbf{B}^{\prime}\right]$ is formed as the direct product of the states of all the blocks.

vi) This iteration continues until the desired length is achieved. At each step the length is $N=2 l+2$ (if [a] consists of one site).

When more than one target state is used, i.e more than one state is wished to be well described, the density matrix is defined as:

$$
\rho_{i i^{\prime}}=\sum_{l} p_{l} \sum_{j} \phi_{l, i j} \phi_{l, i^{\prime} j}
$$

where $p_{l}$ defines the probability of finding the system in the target state $\left|\phi_{l}\right\rangle$ (not necessarily eigenstates of the Hamiltonian).

The method described above is usually called the infinite-system algorithm since the system size increases at each iteration. There is a way to increase precision at each length $N$ called the finite-system algorithm. It consists of fixing the lattice size and zipping to and fro a couple of times until convergence is reached. In this case and for the block configuration [B a a' $\mathbf{B}^{\prime}$ ], $N=$ $l+1+1+l^{\prime}$ where $l$ and $l^{\prime}$ are the number of sites in $B$ and $B^{\prime}$ respectively. In this step the density matrix is used to project onto the left $l+1$ sites (see Fig. 3). In order to keep $N$ fixed, in the next block configuration, the right block $B^{\prime}$ should be defined in $l-1$ sites such that $N=(l+1)+1+1+(l-1)^{\prime}$. The 


\section{System Environment}

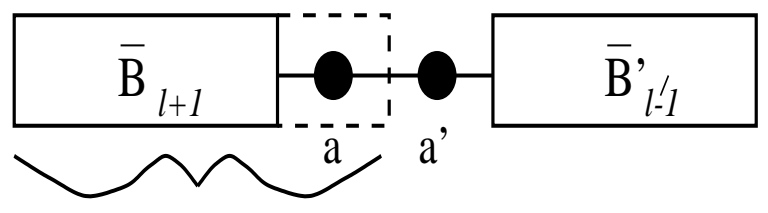

$\mathrm{B}$

$1+2$

Figure 3. One step of the finite-system algorithm, where the total length $N=l+l^{\prime}+2$ is fixed.

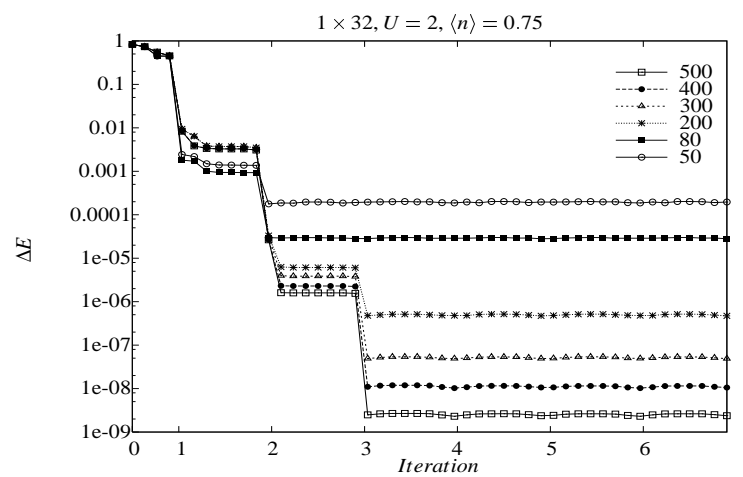

Figure 4. Difference between the ground-state energy obtained from the finite-system DMRG with different number of iterations and number of states kept $m$, and the exact energy calculated using Bethe Ansatz for a 32-site Hubbard model $(U=2$ and filling $n=3 / 4)$. Reprinted from [13] with permission.

operators in this smaller block should be kept from previous iterations (in some cases from the iterations for the system size with $N-2)$ 2]. With this method, a higher precision can be achieved (see Fig. 4). It is only within this finitesystem algorithm that the total errors are controlled (and are proportional) to the discarded error of the density matrix.

As an example of a general DMRG building step let's look at the onedimensional Heisenberg model

$$
H=\sum_{i} \mathbf{S}_{i} \mathbf{S}_{i+1}=S_{i}^{z} S_{i+1}^{z}+\frac{1}{2}\left(S_{i}^{+} S_{i+1}^{-}+S_{i}^{-} S_{i+1}^{+}\right) .
$$

Suppose we want to connect two blocks $\left[B_{1}=\mathbf{B} \mathbf{a}\right]$, defined by states $i, j$, with 
$\left[B_{2}=\mathbf{a}^{\prime} \mathbf{B}^{\prime}\right]$, defined by states $i^{\prime}, j^{\prime}$, to form the Hamiltonian in the superblock, which we will diagonalize to get the energies and eigenstates. Performing an external product between the operators we have stored from previous iterations we have:

$$
\begin{gathered}
{\left[H_{B_{1} B_{2}}\right]_{i j ; i^{\prime} j^{\prime}}=\left[H_{B_{1}}\right]_{i i^{\prime}} \delta_{j j^{\prime}}+\left[H_{B_{2}}\right]_{j j^{\prime}} \delta_{i i^{\prime}}+\left[S_{a}^{z}\right]_{i i^{\prime}}\left[S_{a^{\prime}}^{z}\right]_{j j^{\prime}}} \\
+\frac{1}{2}\left[S_{a}^{+}\right]_{i i^{\prime}}\left[S_{a^{\prime}}^{-}\right]_{j j^{\prime}}+\frac{1}{2}\left[S_{a}^{-}\right]_{i i^{\prime}}\left[S_{a^{\prime}}^{+}\right]_{j j^{\prime}}
\end{gathered}
$$

If fermionic models are to be considered, more operators should be kept, such as, for example, the creation and destruction operators at the borders of the blocks to perform the interblock hopping.

The calculation of static properties like correlation functions is easily done by keeping the operators at each step and performing the corresponding basis change and reduction, in a similar manner as done with the Hamiltonian in each block [4. The energy and measurements are calculated in the superblock.

\subsection{The density matrix projection}

The density matrix leads naturally to the optimal states in the system as we demonstrate below [13, 3].

Let

$$
\left|\psi_{0}\right\rangle=\sum_{i, j=1}^{M} \psi_{i j}|i\rangle|j\rangle
$$

be a state of the universe (system + environment), having real coefficients for simplicity (here we will assume the same configuration of system and environment so $M=M^{\prime}$, but this assumption is not necessary). We want to obtain a variational wave function $\left|\hat{\psi}_{0}\right\rangle$ defined in an optimally reduced space, generated by the $m$ "system" vectors $|\alpha\rangle=\sum_{i=1}^{m} u_{\alpha i}|i\rangle$

$$
\left|\hat{\psi}_{0}\right\rangle=\sum_{\alpha=1}^{m} \sum_{j=1}^{M} a_{\alpha j}|\alpha\rangle|j\rangle
$$

such that the modulus of the difference with the true wave function 5

$$
\left.|| \psi_{0}\right\rangle-\left.\left|\hat{\psi}_{0}\right\rangle\right|^{2}=1-2 \sum_{\alpha i j} \psi_{i j} a_{\alpha j} u_{\alpha i}+\sum_{\alpha j} a_{\alpha j}^{2}
$$


is minimal w.r.t the $a_{\alpha j}$. Setting the derivative in these variables to zero, this condition leads to

$$
\sum_{i} \psi_{i j} u_{\alpha i}=a_{\alpha j}
$$

If we define the density operator for a pure state of the "universe" $\rho=\left|\psi_{0}\right\rangle\left\langle\psi_{0}\right|$, the reduced density matrix of the "system" is:

$$
\rho_{i i^{\prime}}=\sum_{j} \rho_{i j, i^{\prime} j}=\sum_{j}\left\langle j\left|\left\langle i \mid \psi_{0}\right\rangle\left\langle\psi_{0} \mid i^{\prime}\right\rangle\right| j\right\rangle=\sum_{j} \psi_{i j} \psi_{i^{\prime} j}
$$

Replacing 8 in Eq. 7 and using the above expression we obtain the condition:

$$
1-\sum_{\alpha i i^{\prime}} u_{\alpha i} \rho_{i i^{\prime}} u_{\alpha i^{\prime}}=1-\sum_{\alpha=1}^{m} \omega_{\alpha}
$$

where $u_{\alpha i^{\prime}}$ change basis from $|i\rangle$ to $|\alpha\rangle$ and $\omega_{\alpha}$ are the density-matrix eigenvalues. The above expression is minimum when using the largest eigenvalues and corresponding eigenvectors of the density matrix $\rho$, which are all positive or zero. So we have shown that the density matrix leads naturally to the optimal reduced basis giving the best approximation to the initial state. The last term corresponds to the discarded error.

2.1.1 Quantum information interpretation. Another alternative and very interesting interpretation of the density matrix stems from quantum information theory. The DMRG provides an exciting link between strongly correlated systems and quantum information, by providing another perspective into quantum phase transitions and interacting wave functions. As stated by J. Preskill in [20] "The most challenging and interesting problems in quantum dynamics involve understanding the behaviour of strongly-coupled many-body systems[...]. Better ways of characterizing the features of many particle entanglement may lead to new and more effective methods for understanding the dynamical behaviour of complex quantum systems"

In Ref. 21] an interpretation of the correlation functions of systems at criticality is given in terms of wave function entanglement. Recent work 22, 23, 24 has focused on the quantum information perspective of DMRG (see also [25]).

To understand the entanglement between the two parts of a bipartite "universe", say system $\mathrm{S}$ and environment $\mathrm{E}$ one can perform a Schmidt decomposition [26] of the wave function of the "universe" (assuming it is in a pure state), i.e. writing the wave function as a product of states of $\mathrm{S}$ and $\mathrm{E}$ : 


$$
|\psi\rangle=\sum_{\alpha=1}^{\min \left(N_{S}, N_{E}\right)} \sqrt{\omega_{\alpha}}\left|\alpha^{S}\right\rangle\left|\alpha^{E}\right\rangle,
$$

where $N_{S}$ and $N_{E}$ are the Hilbert space sizes of system and environment respectively. Tracing upon the environment we obtain the system density matrix

$$
\rho_{S}=\sum_{\alpha=1}^{\min \left(N_{S}, N_{E}\right)} \omega_{\alpha}\left|\alpha^{S}\right\rangle\left\langle\alpha^{S}\right| .
$$

Similarly, tracing upon the system degrees of freedom, we obtain $\rho_{E}$. Both density matrices have the same rank given by $r \leq \min \left(N_{S}, N_{E}\right)$. One can now affirm that the state $|\psi\rangle$ is entangled if and only if the Schmidt rank $r>1$.

A quantitative measure of entanglement is given by the von Neumann entropy [27,28] defined in either subsystem, (here for example in the subsystem S), by:

$$
S_{S}=-\operatorname{Tr} \rho_{S} \log _{2} \rho_{S}=-\sum_{\alpha} \omega_{\alpha}^{S} \log _{2} \omega_{\alpha}^{S}
$$

By keeping the highest eigenvalues $\omega_{\alpha}^{S}$ of the density matrix, for the general case, one obtains the largest entropy $S$ and, hence, the maximum entanglement between system and environment. It follows from the singular value decomposition theorem that, for a pure target state and any length of system $S$ and environment $E$ blocks, $S_{S}+S_{E}+I=S_{\text {universe }}(=0$ for a pure target or universe state), where $I$ is the mutual information of the blocks and measures the correlation between them. If both blocks are uncorrelated, then $I=0$. It also stems from the above that for periodic boundary conditions, where the density matrix eigenvalues $\omega_{\alpha}$ have a slower decay, the block entropy is also larger than for open boundaries. Therefore, the DMRG will perform better for models or setups which have lower block entropy.

This quantum information perspective leads to an interesting analysis of the performance of the DMRG in different dimensions. The entropy $S$ is proportional to the number of states to be kept in order to maintain the relevant information and it depends on system size $N\left(S_{N}\right)$. Using geometric arguments in a $(\mathrm{d}+1)$-dimensional field theory including a $(\mathrm{d}-1)$-dimensional hypersurface dividing the universe in two $(\mathrm{S}+\mathrm{E})$, it is shown that the entropy, which resides 
essentially at the surface, scales as the hypersurface area 29]

$$
S_{N} \alpha(N / \lambda)^{d-1}
$$

were $\lambda$ is an ultraviolet cutoff. For one dimension $(d=1)$ a more detailed calculation leads to a logarithmic scaling for gapless critical systems having the universal form $S_{N}=c / 3 \ln (N)+\lambda_{1}$ (where $c$ is the central charge of the underlying conformal field theory and $\lambda_{1}$ is related to the ultraviolet cutoff). One also obtains a saturated entropy for non critical, gapped systems [243031] when the system size exceeds the correlation length (which is infinite for critical systems). This shows that for two dimensions, as the number of relevant states to be kept increases with system size, the DMRG isn't an appropriate method as it is conceived. It is, however, quite reliable for sufficiently small systems.

The influence of open boundary conditions on the entropy of entanglement for critical XYZ spin chains was analyzed in [32, finding an additional alternating term connected with the antiferromagnetic nature of the model.

There have been several recent publications exploiting the quantum information perspective of the DMRG. For example, the time dependence of the the block entropy in spin models with sudden changes in the anisotropies was studied in 33. To measure the entanglement between two halves of an anisotropic Heisenberg chain separated by an impurity bond, the dependence of the block entropies (calculated using the density matrix as explained above) with system size and anisotropy was done in [34, finding different behaviours between the antiferro and ferromagnetic cases. The entropy of two sites in a one-dimensional system $S=-\operatorname{Tr} \rho_{i, i+1} \log _{2} \rho_{i, i+1}$ was used as a detector of phase transitions in spin and fermionic systems and applied to models like the bilinear-biquadratic $S=1$ Heisenberg chain, the ionic Hubbard model and the neutral-ionic transition in a donor-acceptor model for molecular chains 35. A similar quantity was used in 36 to calculate the entanglement between molecular orbitals. This two-site method turns out to work better than the single-site entropy for the detection of quantum phase transitions (QPT) proposed in 37. since it comprises non local correlations and can detect phases with off-diagonal long range order. An even better approach seems to be the detection of QPT by using block entropies as proposed in 38 (without resorting to the DMRG) as also shown in [37.

\section{$2.2 \quad$ General remarks}

With respect to accuracy and convergence, the DMRG behaves in different ways depending on the nature of the problem. Its success strongly relies on the existence of the so-called matrix product states MPS, a simple version of which is the AKLT (Affleck-Kennedy-Lieb-Tasaki) state for spin 1 chains [39. 
The DMRG can be viewed as a variational approach within this formulation [40, 41, 23. For cases where the quantum ground state can be represented exactly as a matrix-product state, Östlund and Rommer showed that the renormalization eventually converges to a fixed point and the density matrix has a finite spectrum of non vanishing eigenvalues (see also [42], where a nice example is given based on the non-hermitean $q$-symmetric Heisenberg model using corner transfer matrices). Gapful models also lead to an excellent performance of the method, since the density matrix eigenvalue spectrum decays exponentially and so does the truncated weight with increasing the number of states kept $m$ [44, 43]. Instead, in the case of critical 1D models with algebraically decaying correlation functions, it has been shown that the eigenvalue spectrum decays much slower [45] which slows down further with system size [46]. An analysis of how to circumvent the problems arising near critical points by exploiting conformal field theory predictions using multi-targeted sates is done in [47] and, in [48, a mathematical description of how matrix-product states approximate the exact ground state is given. For two-dimensional systems it can be shown that the eigenvalue decay of the density matrix is extremely slow, thus the method becomes very unreliable for dimensions higher than one [49]. An interesting optimization study in momentum space using concepts of quantum information entropy to select the best state ordering configurations was performed in Ref. 28] and also applied to quantum chemistry calculations. They conclude that the optimum results are obtained when the states with maximum entropy are located at the center of the chain. However, a subsequent study by the same authors led to the conclusion that a criterion based on the entropy profile alone does not lead to optimal ordering [50] and further considerations are given based on quantum data compression concepts. In Ref. 23] the DMRG's low performance with periodic boundary conditions is analyzed and an alternative proposal based on highly entangled states is presented which leads to a much higher precision, comparable to the open boundary conditions results. In a recent work [51, S. White proposes an interesting correction to the DMRG method which consists of including only a single site added to the system, where the subsequent incompleteness of the environment block is compensated by considering a corrected density matrix which takes into account fluctuations of the added site. Comparing this method to the standard two-added-site method he obtains a similar accuracy for open boundary conditions (but faster by a factor of 3) and a much higher accuracy for periodic boundary conditions.

An analytical formulation combining the block renormalization group with variational and Fokker-Planck methods is detailed in [52. The connection of the method with quantum groups and conformal field theory is treated in 53. and an interesting derivation of the reduced density matrix for integrable fermionic and bosonic lattice models from correlation functions was done in 
[54. The articles mentioned above give a deep insight into the essence of the DMRG method.

\section{$2.3 \quad$ Symmetries}

It is crucial to include symmetry in the DMRG algorithm since important reductions in the Hilbert space size can be achieved by fixing the quantum numbers associated with each symmetry. It allows to consider a smaller number of states, enhance precision and obtain eigenstates with definite quantum numbers.

For example, most of the models treated with DMRG conserve the total $z$ spin projection $S_{z}$ and particle number in the whole system, so special care has to be taken when constructing the superblock Hamiltonian, remembering that each block is constituted by several states with different quantum numbers. It is easy to see that when the superblock quantum number can be obtained as an addition of quantum numbers of each constituent part of the system, the density matrix is block diagonal.

Non abelian or non additive symmetries like $\mathrm{SU}(2)$ or total spin are more difficult to implement. Sierra and Nishino showed that Interaction-round-aface (IRF) Hamiltonians represent Hamiltonians with a continuous symmetry and developed a DMRG algorithm appropriate to this model [55. This method was applied to several spin models with excellent performance [55, 56. Total spin conservation, continuous symmetries and parity have been treated also in [57,60, 61, 58, 59] and recently used in Ref. 62 in carbon nanotubes, where flat band ferromagnetism was found.

Contrary to momentum-space DMRG (see Sect 5.1), in real space the total momentum cannot be fixed, because there is no way to fix the corresponding phases in the states that diagonalize the density matrix. However, a very good approximation to having states with fixed momenta can be done by keeping the appropriate target states 63 .

\section{Applications}

Since its development in 1992, the number of papers using DMRG has grown enormously and other improvements to the method have been performed. For example, since 1998 there have been around 80 papers a year using DMRG. There have also been several improvements to the method and it is now used in areas that are very different to the original strongly correlated electron system field. We would like to mention some applications where this method has proved to be useful. Other applications related to further developments of the DMRG will be mentioned in subsequent sections. 
An important benchmark in calculations using DMRG was achieved by White and Huse 64 when calculating the spin gap in a $S=1$ Heisenberg chain obtaining $\Delta=0.41050 J$ with unprecedented accuracy. They also calculated very accurate spin correlation functions and excitation energies for one and several magnon states and performed a very detailed analysis of the excitations for different momenta. They obtained a spin correlation length of 6.03 lattice spacings. Simultaneously Sørensen and Affleck [65] also calculated the structure factor and spin gap for this system up to length 100 with very high accuracy, comparing their results with the nonlinear $\sigma$ model. In a subsequent paper [66] they applied the DMRG to the anisotropic $S=1$ chain, obtaining precise values for the Haldane gap. They also performed a detailed study of the $S=1 / 2$ end excitations in an open chain. Thermodynamical properties in open $S=1$ chains such as specific heat, electron paramagnetic resonance (EPR) and magnetic susceptibility calculated using DMRG gave an excellent fit to experimental data, confirming the existence of free spins $1 / 2$ at the boundaries 67686970 . A related problem, i.e. the effect of non-magnetic impurities in spin systems (dimerized, ladders and 2D) was studied in [71,72. In addition, the study of magnon interactions and magnetization of $S=1$ chains was done in [73, supersymmetric spin chains modelling plateau transitions in the integer quantum Hall effect in 74] and ESR studies in these systems was considered in 75]. For larger integer spins there have also been some studies. Nishiyama and coworkers [76] calculated the low energy spectrum and correlation functions of the $S=2$ antiferromagnetic Heisenberg open chain. They found $S=1$ end excitations (in agreement with the Valence Bond Theory). Edge excitations for other values of $S$ have been studied in Ref. [77]. Almost at the same time Schollwöck and Jolicoeur [78] calculated the spin gap in the same system, up to 350 sites, $(\Delta=0.085 J)$, correlation functions that showed topological order and obtained a spin correlation length of 49 lattice spacings. More recent accurate studies of $S=2$ chains are found in [79, 56, 80, and of $S=1$ chains in staggered magnetic fields 81 including a detailed comparison to the non-linear sigma model in [82. In Ref. 83 the dispersion of the single magnon band and other properties of the $S=2$ antiferromagnetic Heisenberg chains were calculated and the phase diagram of $\mathrm{S}=1$ bosons in $1 \mathrm{D}$ lattices, relevant to recent experiments in optical lattices was studied in 84.

Concerning $S=1 / 2$ systems, DMRG has been crucial for obtaining the logarithmic corrections to the $1 / r$ dependence of the spin-spin correlation functions in the isotropic Heisenberg model [85]. For this, very accurate values for the energy and correlation functions were needed. For $N=100$ sites an error of $10^{-5}$ was achieved keeping $m=150$ states per block, comparing with the exact finite-size Bethe Ansatz results. For this model it was found that the data for the correlation function has a very accurate scaling behaviour and advantage of this was taken to obtain the logarithmic corrections in the thermodynamic 
limit. Other calculations of the spin correlations have been performed for the isotropic [86, 87 and anisotropic cases 88. Luttinger liquid behaviour with magnetic fields have been studied in [89, field-induced gaps and string order parameters in 90 and 91 respectively, anisotropic systems in 92, 93 and the Heisenberg model with a weak link in 94. An analysis of quantum critical points and critical behaviour in spin chains by combining DMRG with finite-size scaling was done in 95. Spin systems in more complex lattices like the Bethe lattice were considered in 96] and the effect of twisted boundary magnetic fields in 97.

Similar calculations have been performed for the $S=3 / 2$ Heisenberg chain 98. In this case a stronger logarithmic correction to the spin correlation function was found. For this model there was interest in obtaining the central charge $c$ to elucidate whether this model corresponds to the same universality class as the $S=1 / 2$ case, where the central charge can be obtained from the finite-size scaling of the energy. Although there have been previous attempts 99, these calculations presented difficulties since they involved also a term $\sim 1 / \ln ^{3} N$. With the DMRG the value $c=1$ was clearly obtained.

In Ref. 100, DMRG was applied to an effective spin Hamiltonian obtained from an $\mathrm{SU}(4)$ spin-orbit critical state in 1D. Other applications were done to enlarged symmetry cases with SU(4) symmetry in order to study coherence in arrays of quantum dots 101, to obtain the phase diagram for 1D spin orbital models 102 and dynamical properties in a magnetic field 103 .

Dimerization and frustration have been considered in Refs. 104, 105, 106, 107, 108, 109, 110, 111, 112] and alternating spin chains in 113.

Several coupled spin chains (ladder models) have been investigated in 114 115, 116, 117, 118, 119, 120, spin ladders with cyclic four-spin exchanges in 121, 122, 123, 124] and Kagome antiferromagnets in [125]. Zigzag spin chains have been considered in [126, 127, 128, spin chains of coupled triangles in [129, 130 131, triangular Ising models in [132 and three-leg spin tubes in [133]. As the DMRG's performance is optimal in open systems, an interesting analysis of the boundary effect on correlation functions is done in 15. Magnetization properties and plateaus for quantum spin chains and ladders [134, 135.136. 137. 138 have also been studied. An interesting review on the applications to some exact and analytical techniques for quantum magnetism in low dimension, including DMRG, is presented in 139.

There has been a great amount of applications to fermionic systems such as 1D Hubbard, ionic Hubbard and t-J models [140, 141, 142, 143, 144, 145, 146, 147, 148, 149, 150, 151, 152, Luttinger liquids with boundaries [153, the Falicov-Kimball model [154, the quasiperiodic Aubry-Andre chain [155] and Fibonacci-Hubbard models [156]. A recent calculation obtains accurate values of the Luttinger-liquid parameter $K_{\rho}$ in Hubbard and spin chains from static correlation functions [157]. It has also been applied to field theory [11 158. The 
method has been very successful for several band Hubbard models [159, 160] and extended Hubbard models modelling chains of $\mathrm{CuO}$ plaquettes 161, Hubbard ladders 162, 163, 164, 165, 166] and t-J ladders [167. Recent calculations in doped Hubbard ladders include the observation of stripes in the 6 -leg ladder for large values of the interaction parameter and not for low values [168, the study of orbital currents and charge density waves in ladders of up to 200 rungs 169 and existence of charge order induced by electron-lattice interaction in coupled ladders 170. Ring exchange on doped two-leg ladders were investigated in [171. Also several coupled chains at different dopings have been considered [172,173] as well as flux phases in these systems [174]. Time reversal symmetry-broken fermionic ladders have been studied in [175] and power laws in spinless-fermion ladders in [176]. Long-range Coulomb interactions in the 1D electron gas and the formation of a Wigner crystal was studied in 177. Several phases including the Wigner crystal, incompressible and compressible liquid states, stripe and pairing phases, have been found using DMRG for 2D electrons in high magnetic fields considering different Landau levels [178, 179, 180. Persistent currents in mesoscopic systems have been considered in 181].

Impurity problems have been studied for example in one- 182 and twoimpurity [183. Kondo systems, in spin chains [184] and in Luttinger Liquids and Hubbard models 185, 186. There have also been applications to Kondo and Anderson lattices [187, 188, 189, 190, 191, 192, 193, 194, 57, 195, 196, Kondo lattices with localized $f^{2}$ configurations [197, the two-channel Kondo lattice on a ladder [198] and on a chain [199], a t-J chain coupled to localized Kondo spins [200] and ferromagnetic Kondo models for manganites [201,202 203,204]. See also Refs. 205, 206] for recent results for the antiferro and ferromagnetic Kondo lattice models.

The DMRG has also been generalized to 1D random and disordered systems, and applied to the random antiferromagnetic and ferromagnetic Heisenberg chains [207, 208, including quasiperiodic exchange modulation 209] and a detailed study of the Haldane [210] and Griffiths phase 211] in these systems. Strongly disordered spin ladders have been considered in 212. It has also been used in disordered Fermi systems such as the spinless model [213, 214]. In particular, the transition from the Fermi glass to the Mott insulator and the strong enhancement of persistent currents in the transition was studied in correlated one-dimensional disordered rings [215. Disorder-induced crossover effects at quantum critical points were studied in [216].

There have been recent applications of the DMRG in nanoscopic devices such as transport properties through quantum dots [217], the study of the influence of interactions in a reservoir on the levels of a quantum dot coupled to it 218] and charge sensing in quantum dots [219]. A very interesting application of the DMRG to study single wall carbon nanotubes, where the 
tube is mapped onto a $1 \mathrm{D}$ chain with longer range interactions (depending on the quirality) can be found in Ref. 220] and the study of edge states in doped nanostructures in 221. Inspired in recent experiments of trapped bosonic atoms 222, a one dimensional Bose gas was studied using ab-initio stochastic simulations at finite temperatures covering the whole range from weak to strong interactions 223 . They used a discretized version of the model and a block factorization of the kinetic energy, using the density matrix to select the most relevant states from each block. Also models for trapped ions have been studied using DMRG, where different quantum phases were obtained [224]. A recent study concerns the colossal electroresistance (due to band bending) through the interface between a metal and a strongly interacting system (in 1D) in the presence of an electric potential obeying the Poisson equation. In this case the use of the finite-size method played an important role in the convergence of the ground state due to the local density-dependent potential. 225]

\section{Bosonic degrees of freedom}

A vast research area in correlated systems concerns bosonic degrees of freedom. For example, phonons play fundamental role in models of currently interesting correlated systems such as high-Tc superconductors, manganites, organic compounds, or nanoscopic systems.

A significant limitation to the DMRG method is that it requires a finite basis and calculations in problems with infinite degrees of freedom per site require a large truncation of the basis states [226]. However, Jeckelmann and White developed a way of including phonons in DMRG calculations by transforming each boson site into several artificial interacting two-state pseudo-sites and then applying DMRG to this interacting system [227] (called the "pseudo-site system"). The proposal is based on the fact that DMRG is much better able to handle several few-states sites than few many-state sites 228. The key idea is to substitute each boson site with $2^{N}$ states into $N$ pseudo-sites with 2 states 229]. They applied this method to the Holstein model for several hundred sites (keeping more than a hundred states per phonon mode) obtaining negligible error. In addition, to date, this method is the most accurate one to determine the ground state energy of the polaron problem (Holstein model with a single electron). For a recent comprehensive review on exact numerical methods in electron-phonon problems see [230.

This method has been applied recently to the calculation of pairing, CDW and SDW correlations in the Holstein-Hubbard model with competing electron-electron and electron-phonon interactions, finding an enhanced superconducting exponent when next-nearest neighbour hopping is added [231]. 


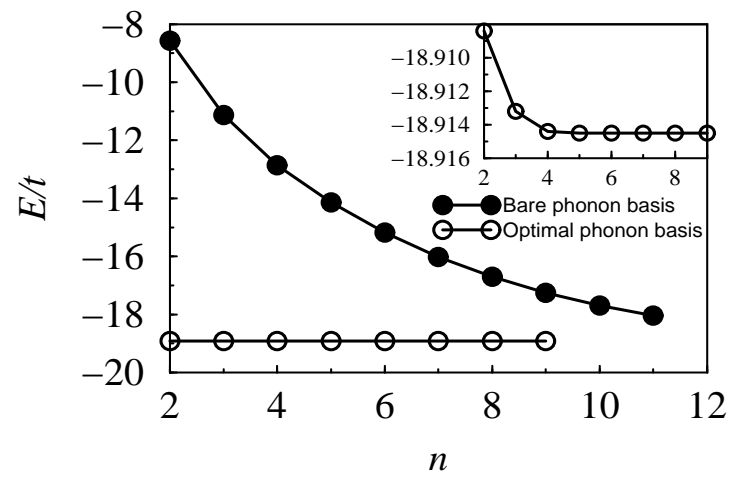

Figure 5. Ground-state energy of a 4 site, half-filled Holstein system vs. the number of phonon states kept on each site of the lattice, for the optimal and bare phonon basis. Reprinted from 232 with permission.

They propose here a way to improve the bare repulsion felt by the electrons in the newly added pseudo-sites in the infinite-size algorithm by adding a chemical potential to these sites, finding lower ground state energies. An interesting result was obtained in 233] using this method combined with the dynamical DMRG (see Sect 8.1) to calculate single-particle spectra in one-dimensional Mott insulators (the Holstein-Hubbard model at half filling in this case). They observe that the charge-spin separation characteristic of $1 \mathrm{D}$ systems is robust against electron-phonon coupling.

An alternative method (the "Optimal phonon basis") 232 is a procedure for generating a controlled truncation of a large Hilbert space, which allows the use of a very small optimal basis without significant loss of accuracy. The system here consists of only one site and the environment has several sites, both having electronic and phononic degrees of freedom. The density matrix is used to trace out the degrees of freedom of the environment and extract the most relevant states of the site in question. In following steps, more bare phonons are included to the optimal basis obtained in this way. This method was successfully applied to study the Holstein model (Fig (5), the interactions induced by quantum fluctuations in quantum strings as an application to cuprate stripes [234, the dissipative two-state system [235] and, recently, the spin-Peierls model [236]. A variant of this scheme is the "four block method", as described in [237. They obtain very accurately the Luttinger liquid-CDW insulator transition in the 1D Holstein model for spinless fermions.

The method has also been applied to pure bosonic systems such as the disordered bosonic Hubbard model [238], where gaps, correlation functions and superfluid density are obtained. The phase diagram for the non-disordered 
Bose-Hubbard model, showing a reentrance of the superfluid phase into the insulating phase was calculated in Ref. 239. It has also been used to study a chain of oscillators with optical phonon spectrum 240 and optical phonons in the quarter-filled Hubbard model for organic conductors [241].

\section{Generalized DMRG}

Since its development, the DMRG has been widely improved in several directions in order to deal with different kinds of problems. It has been generalized to treat models where a representation in momentum or energy space is more appropriate. Based on these ideas, an important extension towards Quantum Chemistry and the calculation of physical properties of small grains and nuclei was performed. Below we describe each of these ideas.

\subsection{Momentum representation}

In 1996, Xiang 242] proposed an alternative formulation of the DMRG in momentum space, rather than in real space, which can be used also in two or more dimensions, it deals better with periodic boundary conditions and has the advantage that the total momentum quantum number can be fixed. This algorithm has inspired the extension of the DMRG to finite fermionic systems, small grains and nuclear physics. The main idea is to write the Hamiltonian in momentum space. If the original model is long ranged, as with Coulomb interactions, then it will turn local in the reciprocal space and the DMRG could be used in the normal way, where now each "site" is a state with definite momentum. The most convenient growth procedure for real space short-ranged Hamiltonians is to start using one-particle states lying near the Fermi energy, above and below, and subsequently add additional more distant states. For longer ranger models other orderings are to be considered [28].

In his original work, Xiang applied these ideas to the 1D and 2D Hubbard models with nearest-neighbour interaction, defined in general as:

$$
H=-t \sum_{<i j>\sigma}\left(c_{i \sigma}^{\dagger} c_{j \sigma}+c_{j \sigma}^{\dagger} c_{i \sigma}\right)+U \sum_{i} n_{i \uparrow} n_{i \downarrow}
$$

The operator $c_{i \sigma}^{\dagger}\left(c_{i \sigma}\right)$ creates (annihilates) an electron on site $i$ with spin projection $\sigma, n_{i \sigma}=c_{i \sigma}^{\dagger} c_{i \sigma}, t$ is the hopping parameter and $U$ the on-site Coulomb interaction.

In momentum space it reads: 


$$
H=\sum_{\mathbf{k} \sigma} \epsilon_{\mathbf{k}} n_{\mathbf{k} \sigma}+\frac{U}{N} \sum_{\mathbf{p}, \mathbf{k}, \mathbf{q}} c_{\mathbf{p}-\mathbf{q} \uparrow}^{\dagger} c_{\mathbf{k}+\mathbf{q} \downarrow}^{\dagger} c_{\mathbf{k} \downarrow} c_{\mathbf{p} \uparrow}
$$

where $n_{\mathbf{k} \sigma}=c_{\mathbf{k} \sigma}^{\dagger} c_{\mathbf{k} \sigma}$ is the number operator for particles with spin $\sigma$ and momentum $\mathbf{k}$ and $\epsilon_{\mathbf{k}}$ is the energy dispersion of the particles.

By defining appropriate operator products using the original ones, the number of operators to be kept can be greatly reduced and the convergence becomes faster and more accurate. Other authors [243, 28] have improved the method and performed more systematic and interesting analysis on the order of states to be kept, depending on the kind of interactions in the model and very accurate dispersion relations have been obtained in 173. The method has a better performance than the real space DMRG in two dimensions for low values of the interaction (as seen in $4 \times 4$ lattice calculations), the accuracy of the method becoming worse for larger values of $U$. Recently, an interesting algorithm for the calculation of eigenstates with definite momentum has been put forward in 244, based on the PEPS (projected entangled pair states). In this case additional degrees of freedom are added for each site and the particular momentum quantum number is fixed by constructing a "projected entangled-multiparticle state" (PEMS). It has been successfully applied to calculate excitation spectra in the bilinear-biquadratic $S=1$ Heisenberg model.

\section{$5.2 \quad$ Grains, nuclei and high energy physics}

Another successful extension to the DMRG is the calculation of physical properties of small fermionic systems, like superconducting grains and models for nuclei. This was developed by Dukelsky, Sierra and collaborators 245. For a review on these applications see the recent publication [246].

A typical and general Hamiltonian for these finite systems consists of oneand two-particle terms of the form

$$
H=\sum_{i j} T_{i j} c_{i}^{\dagger} c_{j}+\sum_{i j k l} V_{i j k l} c_{i}^{\dagger} c_{j}^{\dagger} c_{l} c_{k}
$$

where $T_{i j}$ represents the kinetic energy and $V_{i j k l}$ the residual two-body interaction between the effective particles.

Starting from the single particle levels and a certain filling which defines the Fermi energy, one can define particle and hole blocks in a similar manner as the real space DMRG (see Fig. [6). The empty and occupied levels are the 


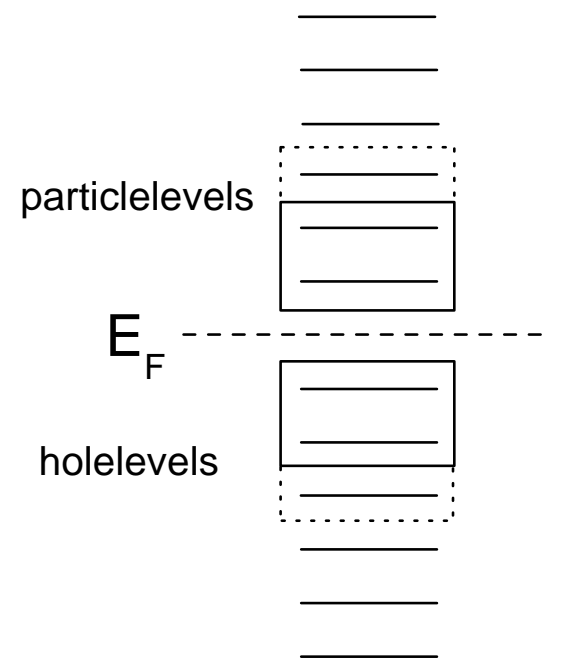

Figure 6. DMRG method for finite fermionic systems where the added "sites" lie successively at larger distances from the Fermi energy.

particle and hole levels respectively (referring to the character of the singleparticle excitations). By successively adding levels at increasing distance from the Fermi level, the DMRG renormalization is applied. This might not be the appropriate level ordering for DMRG for more complex Hamiltonians [28,246].

Within this scheme, superconductivity in small grains has been studied using the half-filled reduced BCS Hamiltonian

$$
H=\sum_{j=1}^{\Omega} \sum_{\sigma= \pm}\left(\epsilon_{j}-\mu\right) c_{j \sigma}^{\dagger} c_{j \sigma}-\lambda d \sum_{i, j=1}^{\Omega} c_{i+}^{\dagger} c_{i-}^{\dagger} c_{j-} c_{j+}
$$

where $i, j=1,2, \ldots, \Omega$ label the single-particle energy levels, $c_{j \sigma}$ are the electron annihilation operators associated with the two time-reversed states $|j, \sigma=+\rangle$ and $|j, \sigma=-\rangle, \mu$ is the chemical potential, $\lambda$ is an adimensional BCS coupling constant and $d$ is the single-particle energy level spacing (assumed to be constant). By using DMRG a smooth superconducting-normal state crossover with decreasing system size was found, a more reliable result than the abrupt transition obtained using exact diagonalization and BCS approximation. This method was also used for two superconducting grains coupled by tunneling 247.

Other recent applications have been in nuclear shell model calculations where a two-level pairing and pairing plus quadrupole interactions model have been considered [248. Here the nucleus is modelled by completely filled core 
shells and valence orbitals partially filled with protons and neutrons where the starting states are the Hartree Fock functions. A realistic calculation for

the ${ }^{24} \mathrm{Mg}$ nucleus showed very slow convergence mainly due to the lack of improvement of the finite-system algorithm in this basis and the fact that total angular momentum cannot be explicitly fixed. A recent work obtained the $s d$ - and $f p$-shell models for ${ }^{28} \mathrm{Si}$ and ${ }^{56} \mathrm{Ni}$ respectively in a different basis using proton-neutron wave function factorization which allows fixing total angular momentum plus the finite-system algorithm 249. The DMRG has also been extended to study complex-symmetric density matrices which describe many-body open quantum systems (where resonant and non-resonant levels are considered) and applied to the unbound nucleus ${ }^{7} \mathrm{He}$ with possible further applications to open quantum dots, open microwave resonators, etc. 250.

A very interesting and successful application is a recent work in High Energy Physics [251]. Here the DMRG is used in an asymptotically free model with bound states, a toy model for quantum chromodynamics, namely the two dimensional delta-function potential. For this case an algorithm similar to the momentum space DMRG 242] was used where the block and environment consist of low and high energy states respectively. The results obtained here are much more accurate than with the similarity renormalization group [252] and a generalization to field-theoretical models is proposed based on the discreet light-cone quantization in momentum space [253].

\subsection{Molecules and Quantum Chemistry}

Using the standard real space DMRG, there have been several applications to molecules and polymers, such as the Pariser-Parr-Pople (PPP) Hamiltonian for a cyclic polyene [254,255] (where long-range interactions are included), magnetic Keplerate molecules [256], molecular Iron rings [257, spin crossover molecules [258] and polyacenes considering long range interactions [259]. The application to conjugated organic polymers was performed by adapting the DMRG to take into account the most important symmetries in order to obtain the desired excited states 60. Also conjugated one-dimensional semiconductors 260. have been studied, in which the standard approach can be extended to complex 1D oligomers where the fundamental repeat is not just one or two atoms, but a complex molecular building block. Relatively new fields of application are the calculation of dynamical properties in the Rubinstein-Duke model for reptons [261] and excitons in dendrimer molecules [262].

Another very important problem is the $a b$ initio calculation of electronic states in Quantum Chemistry. The standard approaches consist of the Density Functional Theory and the Hartree Fock starting point to calculate complex electronic structure (using for example the Configuration Interaction approach). When electron interactions are included, relatively small clusters can 


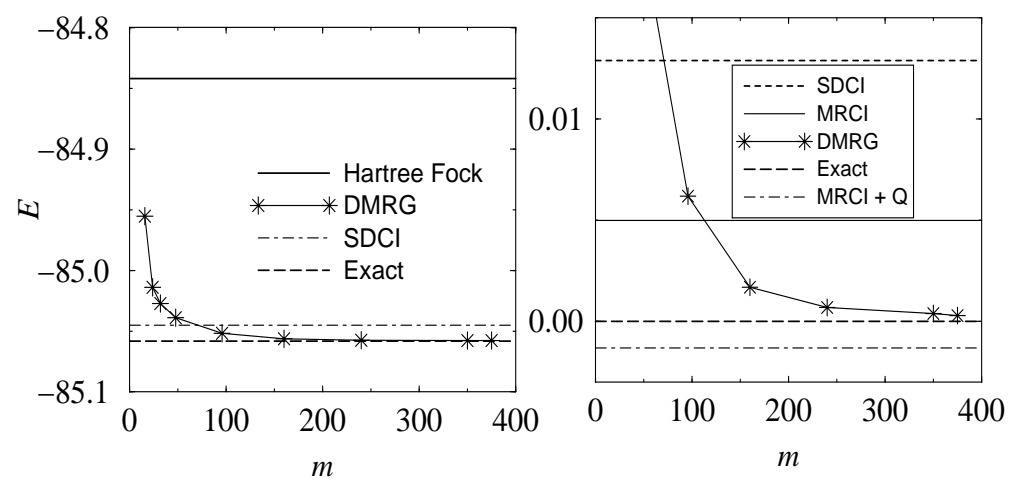

Figure 7. Ground state energy (left) and error (right) of a water molecule in a 25 orbital basis using several methods. The DMRG results are plotted as a funtion of the number of states kept, $m$ (not relevant for the other energies). Reprinted from 263 with permission.

be considered due to the large Hilbert spaces involved. To be able to treat larger molecules, in 1999, White and Martin 263 proposed a way of using the DMRG for quantum chemistry calculations, based on the momentum representation DMRG 242. They considered a general model for molecules, Eq (17), where the electron-nucleus interaction in considered in the first term and the operators indices represent orbitals in the Hartree Fock basis (see also 264,265, 266]). Here, DMRG is applied within the conventional quantum chemical framework of a finite basis set with non-orthogonal basis functions centered on each atom. After the standard Hartree-Fock (HF) calculation in which a Hamiltonian is produced within the orthogonal HF basis, DMRG is used to include correlations beyond HF, where each orbital is treated as a "site" in a 1D lattice. One important difference with standard DMRG is that, as the interactions are long-ranged, several operators must be kept, making the calculation somewhat cumbersome. However, very accurate results have been obtained in a check performed in a water molecule (keeping up to 25 orbitals and $m \simeq 200$ states per block), obtaining an offset of $2.410^{-4}$ Hartrees with respect to the exact ground state energy [267, a better performance than any other approximate method [263] (see Fig[7).

In order to avoid the non-locality introduced in the treatment explained above, White introduced the concept of orthlets, local, orthogonal and compact wave functions that allow prior knowledge about singularities to be incorporated into the basis and an adequate resolution for the cores [264]. The most relevant functions in this basis are chosen via the density matrix. An application based on the combination with the momentum version of DMRG is used in [265] to calculate the ground state of several molecules. In Ref. [268], 
the efficiency of the DMRG method was analyzed. Although it works very well for some molecules, like $\mathrm{Be}_{2}, \mathrm{HF}, \mathrm{H}_{2} \mathrm{O}, \mathrm{CH}_{4}$, calculations in the $\mathrm{N}_{2}$ molecule were not satisfactory. The reasons for the failure could be related to the level ordering taken for the DMRG algorithm, although it is not conclusive. A very careful analysis of the method presented in a pedagogical way can be found in Ref. 269.

In a recent work, quantum information theory concepts were used together with DMRG to calculate orbital interaction in different molecules, such as LiF, $\mathrm{CO}, \mathrm{N}_{2}$ and $F_{2}$ [36. Here the interaction between electrons in two orbitals in a many-body wave function was calculated as the difference between the entanglement of both orbitals taken together and individually (always belonging to the same DMRG "system") for a given wave function. An interesting analysis of the relevance of the level ordering within the DMRG growing procedure is given.

\section{$6 \quad$ Higher dimensions $(D>1)$}

The possibility of using DMRG in two dimensions is currently one of the most challenging problems. As we mentioned above equation 14), due to the increasing number of states to be kept in dimensions higher than one, the DMRG is not suited for these cases. By analyzing analytically the spectra of a two-dimensional system of coupled oscillators, Chung and Peschel 49. showed that the density matrix eigenvalues decay much slower than in the one-dimensional case. However, if the system size is kept relatively small, in two dimensions one can reach larger systems than with exact diagonalization methods and the zero temperature results are more reliable than with Monte Carlo methods. The first attempts to extend the DMRG to two dimensions considered different one-dimensional kind of paths like the one shown in figure 8. where the DMRG is used in the standard way. The main drawback of this method is that many operators have to be kept and the interactions are longranged in this $1 \mathrm{D}$ mapping.

Quite large quasi-2D systems can be reached, for example in 270] where a 4x20 lattice was considered to study ferromagnetism in the infinite-U Hubbard model; the ground state of a 4-leg t-J ladder in 271]; the one- and two-hole ground states in $9 \mathrm{x} 9$ and 10x7 t-J lattices in [272]; a doped 3-leg t-J ladder in [273]; the study of striped phases in 274]; domain walls in 19x8 t-J systems in 275] the 2D t-J model in 276] and the magnetic polaron in a 9x9 t-J lattice in [277]. Also big $\mathrm{CaV}_{4} \mathrm{O}_{9}$ spin-1/2 lattices reaching $24 \times 11$ sites [18] have been studied as well as a recent study of the spin liquid phase in the anisotropic triangular Heisenberg model in up to 8x18 lattices [278].

There have also been some recent alternative attempts to implement DMRG 


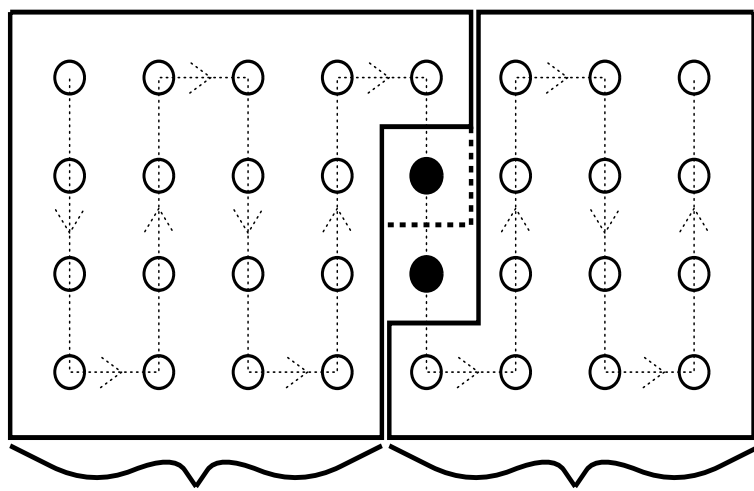

system environment

Figure 8. General sweeping and superblock configuration for a two-dimensional lattice (from [13], with permission)

in two and higher dimensions with clever block arrangements 279, 243, 280, 281, 282, 283 but the performances are still poorer than in 1D. A recent extension using a novel DMRG algorithm for highly anisotropic spin systems has shown promising results [284]. Here a two-step method is proposed which is based on the diagonalization of a single chain to obtain the lowest-lying states and then using DMRG to couple these chains in a 1D manner. As long as the energy width of the retained states is much larger than the interchain coupling, this method yiels results which are comparable to Quantum Monte Carlo calculations.

Another problem in physics that has been handled using DMRG is that of electrons in a high magnetic field. Shibata and Yoshioka 178 179 180] used the eigenstates of free electrons in a perpendicular magnetic field in the Landau gauge to represent the local orbitals. By choosing these single particle states as effective sites, where the wave functions are parametrized by only two quantum numbers (the Landau level and the $x$ component of the center of coordinates of an electron in a cyclotron motion), it provides a natural mapping onto a $1 \mathrm{D}$ system (see also [285]). The long-range Coulomb interactions between the electrons stabilizes various different electronic states depending on the filling of the Landau levels. If the magnetic field is larger than the characteristic Coulomb interactions, the electrons in filled Landau levels can be considered inert and the ground state is determined by the partially filled level. The ground state properties for different filling factors were characterized using 
the expectation value of the pair correlation function

$$
g(\mathbf{r}) \equiv \frac{L_{x} L_{y}}{N_{e}\left(N_{e}-1\right)}\left\langle\Psi\left|\sum_{i \neq j} \delta\left(\mathbf{r}+\mathbf{R}_{i}-\mathbf{R}_{j}\right)\right| \Psi\right\rangle
$$

where $\mathbf{R}_{i}$ is the center coordinate of the $i$ th electron's wave function, $\mathbf{r}$ is the relative distance between the pair of electrons and $L_{x, y}$ are the unit cell dimensions. In 178 the phase diagram for the third Landau level was obtained, showing a Wigner crystal for low filling factors, up to $\nu=0.24$, where a 2electron bubble phase forms. For $\nu>0.38$, a striped phase shows up (see Fig. 9) and in 180 an analysis of the excitonic behaviour of the $\nu=1$ bilayer quantum Hall systems was done.

(a) Wigner crystal

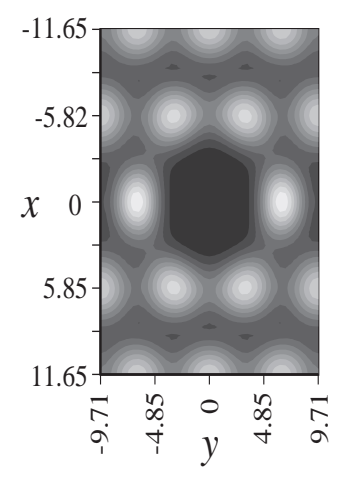

(b) two-electron bubble

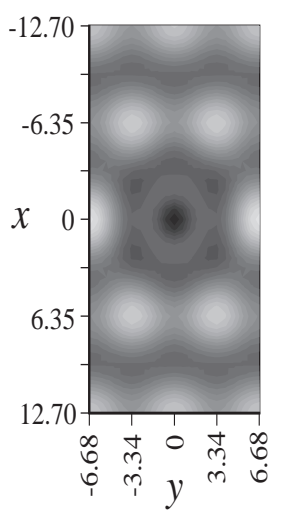

(c) stripe

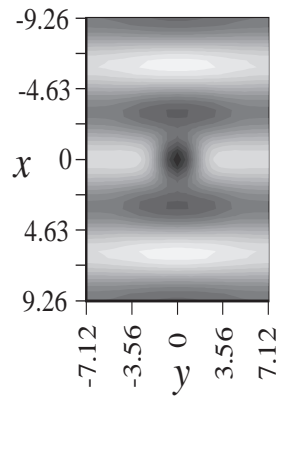

Figure 9. Pair correlation function of a) a striped state, b) a two-electron bubble state and c) a Wigner crystal (from 285, with permission)

A very promising approach towards two dimensional calculations was recently proposed by Verstraete and Cirac [22, based on the projected entangledpair-states (PEPS). It relies on the matrix-product states (MPS) approach to describe the variational wave functions which is the basis of success of the DMRG. Following Ref. 222's notation, let's represent the 1D state $|\Psi\rangle$ as a MPS of $N d$-dimensional physical systems $|s\rangle$ (e.g. $d=2$ for individual $S=1 / 2$ spins). We then enlarge the Hilbert space by replacing each physical site $|s\rangle$ by two auxiliary systems $a_{k}$ and $b_{k}$ each of a certain dimension $D$ (to be obtained variationally to minimize the energy), except for the extremes of the chain, where only one auxiliary system is added, and set them in the highest entangled state $|\phi\rangle=\sum_{n=1}^{D}|n, n\rangle$ (see Fig. 10). Each of these states is then 
(b) (a) $\odot-0-6-0$

(c)

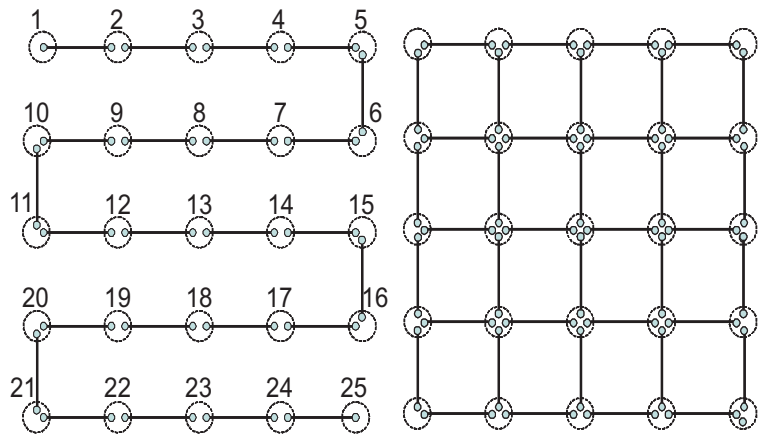

Figure 10. Schematic representation of MPS (a) 1 dimension and (b) 2 dimensions. (c) representation of 2D PEPS. The bonds represent pairs of maximally entangled D-dimensional auxiliary spins and the circles denote projectors that map the auxiliary spins to the physical ones. Reproduced from [22] with permission.

projected to the real spin by applying an operator $Q_{k}$ to each pair to obtain the real entangled system,

$$
\begin{aligned}
|\Psi\rangle & =\quad Q_{1} \otimes Q_{2} \otimes \ldots Q_{N}|\phi\rangle \ldots|\phi\rangle \\
& =\sum_{s_{1}, \ldots, s_{N}=1}^{d} \mathrm{~F}_{1}\left(A_{1}^{s_{1}}, \ldots, A_{N}^{s_{N}}\right)\left|s_{1}, \ldots, s_{N}\right\rangle .
\end{aligned}
$$

Here the matrices $A_{k}^{s}$ have elements $\left(A_{k}^{s}\right)_{l, r}=\left\langle s\left|Q_{k}\right| l, r\right\rangle$ and the indices $l$ and $r$ of each matrix $A_{k}^{s}$ are related to the left and right bonds of the auxiliary systems with their neighbors. The function $\mathrm{F}_{1}$ denotes the trace of the product of the matrices. An extension of this state was proposed for two dimensions (see Fig. 10k), where now each site is represented as the projection of $4 \mathrm{D}$ dimensional auxiliary systems. The ground state of a Hamiltonian corresponds to the PEPS with a given dimension $D$ which minimizes the energy and this is performed by iteratively optimizing each $A$ tensor while keeping the others fixed, in a similar manner as the finite-size DMRG zipping procedure. Within this MPS picture, it was shown also by Verstraete and collaborators [286] that a uniform picture can be given of Wilson's numerical renormalization group method [8] together with White's DMRG. 


\section{Time-dependent analysis}

One of the most important recent developments of the DMRG concerns realtime calculations: the so-called time-dependent DMRG. Its potential applications include the analysis of the evolution of wave functions, the calculation of transport properties in low-dimensional systems, non-equilibrium transport through nanosystems or dissipative quantum mechanics. A recent comprehensive review on the time-dependent DMRG can be found in Ref. 287.

There are two main different schemes to tackle time-dependent problems. Both different approaches have been classified as dynamic (Hilbert space) and adaptive time-dependent methods. In the first case the Hilbert space for the initial wave function is kept as large as possible so as to be able to describe the time-evolved state for sufficiently long times. In the second approach, instead, the states kept are calculated at each time interval, adapting to the evolving Hamiltonian.

The first method was developed by Cazalilla and Marston [288] in a simplified version which targeted only the ground state (defined as static method in 287]). After growing the system up to a certain size, a quantum dot or a junction is added and a time-dependent perturbation is $H^{\prime}(t)$ set on. The time-dependent Schrödinger equation is then integrated forward in time using a discretized algorithm:

$$
i \hbar \frac{\partial}{\partial t}|\Psi(t)\rangle=\left[\left(H_{\text {trunc }}-E_{0}\right)+H^{\prime}(t)\right]|\Psi(t)\rangle .
$$

where the initial state is chosen to be the ground state of the unperturbed system. As this method does not conserve unitarity, the alternative CrankNicholson procedure [289],

$$
|\Psi(t+d t)\rangle \simeq \frac{1-i H_{\text {trunc }}(t) \delta t / 2}{1+i H_{\text {trunc }}(t) \delta t / 2}|\Psi(t)\rangle
$$

can be used. The calculation of the denominator can be calculated using the biconjugate gradient approach [290. If more than one target state is used during the growing procedure, a higher precision is obtained and the time evolution can be followed longer [291]. Fig. 11] shows the current through two quantum systems calculated using this method.

A slightly different approach was used by Schmitteckert [292]. Instead of using the differential equation, he applied the time evolution operator $\exp (-i H t)$ directly by using a Krylov basis expansion of the matrix exponential, using a Lanczos procedure, within the finite-system algorithm. Using this method, and keeping information of the evolved wave function in a similar manner as 


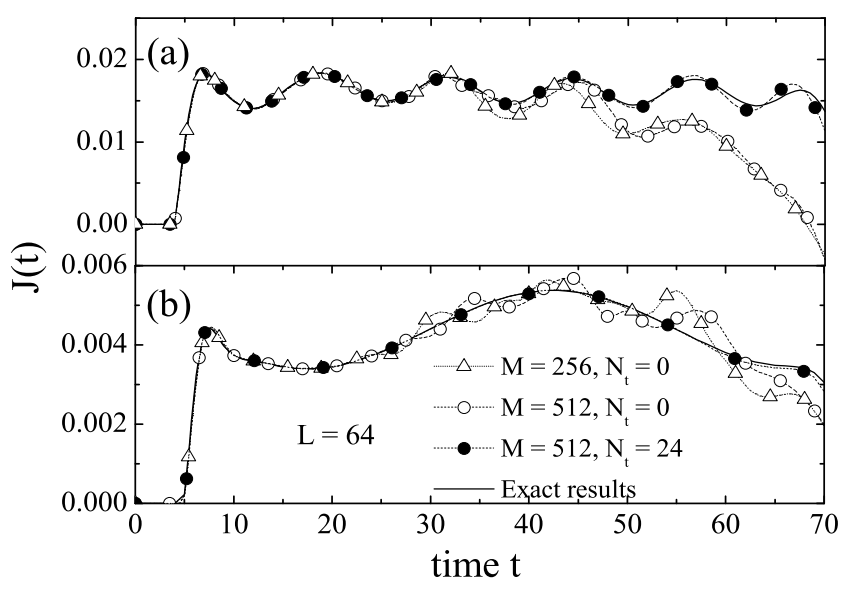

Figure 11. Current through a) a non-interacting quantum dot and b) a junction between two leads (defined in 288). $N_{t}$ is the number of excited target states, $L$ the number of sites and $M$ the number of states kept (reprinted with permission from 291).

described below, transport properties of spinless electrons through strongly interacting 1D systems with arbitrary bias was calculated 293. This method does not involve Trotter approximations (see below) and is appropriate to deal with general geometries and interactions. An improvement to this $d y$ namic method was performed in [294] by using the 4th order Runge-Kutta method and targeting a small interval of time (time-step targeted method).

The adaptive approach [295, 290] was based on the algorithm developed by Vidal for the time evolution of weakly entangled quantum states [296, originally formulated in the matrix product language.

For a one dimensional system with nearest-neighbour interactions, the infinitesimal time evolution operator $\exp (-i H d t)$ can be decomposed using a second order (of course higher orders can also bee applied) Trotter-Suzuki expansion into link operators (to order $d t^{3}$ ) as:

$$
e^{-i d t H} \approx e^{-i d t H_{1} / 2} e^{-i d t H_{2} / 2} \ldots e^{-i d t H_{2} / 2} e^{-i d t H_{1} / 2},
$$

where $H_{i}$ is the Hamiltonian term acting on link $i$. When applied $t / d t$ times to get one time interval, the error is of the order $\left(d t^{2}\right) t$, so, in the worst case it scales linearly with $t$ (an extensive error analysis is performed in [297]).

One important characteristic of the DMRG is that, when operators are cal- 
culated acting on the single sites outside the renormalized blocks, the result is exact. Taking advantage of this, the above decomposition can be applied sequentially on these exact sites, in a finite-sweep-like procedure. This algorithm can be easily incorporated into a static DMRG code. Using this method, White and Feiguin 295] calculated the same transport properties as in Fig. 11 and their results match the exact result (full line) very precisely for larger times. They have also calculated other time-dependent responses like the dispersion of a spin excitation in a 200-sites spin-1 Heisenberg chain (see Fig. 12).

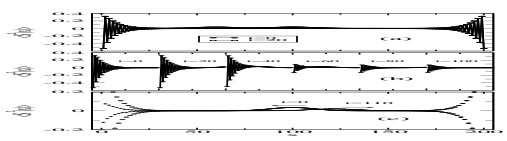

Figure 12. Time evolution of a spin excitation wave-packet in a spin-1 Heisenberg chain with 200 sites. In b) only the 50 leftmost sites are shown. Reprinted from 295] with permission.

The results obtained by this method are very accurate thus allowing to obtain frequency-dependent quantities by Fourier transformation 295. Some recent applications include the calculation of the evolution of one-dimensional density waves 298] and the dynamics of the superfluid-to-Mott transition [299] (here using the matrix formalism [296]) in ultracold bosons in an optical lattice and the analysis of charge spin separation in cold Fermi gases [300] by observ- 
ing the time evolution of wave packets 301. It was also applied to calculate the Zener breakdown in band and Mott insulators 302, real-time dynamics of spin 1/2 Heisenberg chains [297, conductance through strongly correlated systems 303 and a single atom transistor in 1D optical lattice 304. An interesting application of this time-dependent analysis is the recent study of the evolution of the entropy of a block of spins in the Heisenberg chain after a sudden quench in the anisotropy [33, which gives information of the propagation of entanglement and critical behaviour.

This method is very precise and easy to implement. However, it has the disadvantage of being restricted by geometry and nearest-neighbour interactions. For other geometries the dynamic method mentioned above is preferred.

\section{Zero temperature dynamics}

DMRG can also be used to calculate dynamical properties of low-dimensional systems, useful to interpret experimental results from, for example, nuclear magnetic resonance (NMR), neutron scattering, optical absorption and photoemission, among others. There have been two main approaches to the dynamics: the Lanczos [63, 305] and correction vector techniques [305, 306, 307. The first gives complete information of the whole excitation spectrum at the expense of less accuracy for large systems, specially at high energies. The latter, instead, focuses on particular energy values and gives more precise information, being numerically much more expensive.

\subsection{Lanczos technique}

We want to calculate the following dynamical correlation function at $T=0$ :

$$
C_{A}\left(t-t^{\prime}\right)=\left\langle\psi_{0}\left|A^{\dagger}(t) A\left(t^{\prime}\right)\right| \psi_{0}\right\rangle
$$

where $A^{\dagger}$ is the Hermitean conjugate of the operator $A, A(t)$ is the Heisenberg representation of $A$, and $\left|\psi_{0}\right\rangle$ is the ground state of the system. Its Fourier transform is:

$$
C_{A}(\omega)=\sum_{n}\left|\left\langle\psi_{n}|A| \psi_{0}\right\rangle\right|^{2} \delta\left(\omega-\left(E_{n}-E_{0}\right)\right)
$$

where the summation is taken over all the eigenstates $\left|\psi_{n}\right\rangle$ of the Hamiltonian $H$ with energy $E_{n}$, and $E_{0}$ is the ground state energy. 
Defining the Green's function

$$
G_{A}(z)=\left\langle\psi_{0}\left|A^{\dagger}(z-H)^{-1} A\right| \psi_{0}\right\rangle
$$

the correlation function $C_{A}(\omega)$ can be obtained as

$$
C_{A}(\omega)=-\frac{1}{\pi} \lim _{\eta \rightarrow 0^{+}} \operatorname{Im} G_{A}\left(\omega+i \eta+E_{0}\right)
$$

The function $G_{A}$ can be written in the form of a continued fraction:

$$
G_{A}(z)=\frac{\left\langle\psi_{0}\left|A^{\dagger} A\right| \psi_{0}\right\rangle}{z-a_{0}-\frac{b_{1}^{2}}{z-a_{1}-\frac{b_{2}^{2}}{z-\ldots}}}
$$

The coefficients $a_{n}$ and $b_{n}$ can be obtained using the following recursion equations 308, 309]:

$$
\left|f_{n+1}\right\rangle=H\left|f_{n}\right\rangle-a_{n}\left|f_{n}\right\rangle-b_{n}^{2}\left|f_{n-1}\right\rangle
$$

where

$$
\begin{aligned}
\left|f_{0}\right\rangle & =A\left|\psi_{0}\right\rangle \\
a_{n} & =\left\langle f_{n}|H| f_{n}\right\rangle /\left\langle f_{n} \mid f_{n}\right\rangle, \\
b_{n}^{2} & =\left\langle f_{n} \mid f_{n}\right\rangle /\left\langle f_{n-1} \mid f_{n-1}\right\rangle ; \quad b_{0}=0
\end{aligned}
$$

For finite systems the Green's function $G_{A}(z)$ has a finite number of poles. Within this formulation it is not necassary to compute all coefficients $a_{n}$ and $b_{n}$ since the spectrum is nearly converged already with the first few tens or hundreds of them, depending on the problem. The DMRG technique presents a good framework to calculate such quantities. With it, the ground state, Hamiltonian and the operator $A$ required for the evaluation of $C_{A}(\omega)$ are obtained. An important requirement is that the reduced Hilbert space should also describe with great precision the relevant excited states $\left|\psi_{n}\right\rangle$. This is achieved by choosing the appropriate target states. For most systems it is enough to consider as target states the ground state $\left|\psi_{0}\right\rangle$ and the first few $\left|f_{n}\right\rangle$ with $n=0,1 \ldots$ and $\left|f_{0}\right\rangle=A\left|\psi_{0}\right\rangle$ as described above. In doing so, states in the reduced Hilbert space relevant to the excited states connected to the ground state via the operator of interest $A$ are included. The fact that $\left|f_{0}\right\rangle$ is an excellent trial state, in particular, for the lowest triplet excitations of the twodimensional antiferromagnet was shown in Ref. [310]. Of course, if the number 


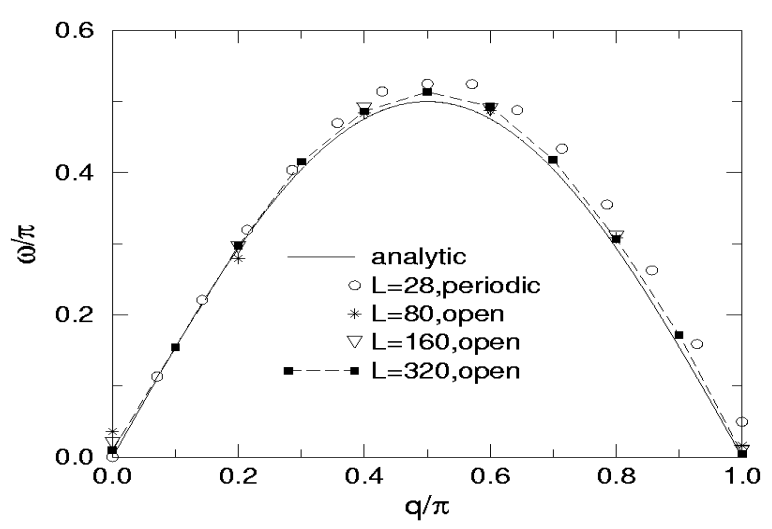

Figure 13. Lower bound of the continuous excitation band of the AF $S=1 / 2$ Heisenberg model for different system sizes $L$ (reprinted from Ref. [305] with permission). The full line is the analytical result for the infinite system using Bethe Ansatz.

$m$ of states kept per block is fixed, the more target states considered, the less precisely each one of them is described. An optimal number of target states and $m$ have to be found for each case. Due to this reduction, the algorithm can be applied up to certain lengths, depending on the states involved. For longer chains, the higher energy excitations will become inaccurate. Proper sum rules have to be calculated to determine the errors in each case.

This method has been successfully applied to a number of problems, like spin 1/2 (see Fig [13), 3/2 [85, 98, 63, 305] and spin 1 chains [305], the spin-boson model [311], the Holstein model [312,233] and spin-orbital chains in external fields 313. It was also applied to extract spin-chain dispersion relations 314, dynamics of spin ladders [123], spectral functions in the infinite-U Hubbard model [141, optical response and spinon and holon excitations in 1D Mott insulators 315 316. In a recent work, this method, together with the correction vector described below, was used to calculate the spectral function of ultrasmall Kondo systems and analyzed finite-size and even-odd effects 317] with great accuracy. In Section 10 we will describe its application as the impurity solver within the Dynamical Mean Field Theory method (DMFT). 


\subsection{Correction-vector method}

This method focuses on a particular energy or energy window, allowing for a more precise description in that range and the possibility of calculating spectra for higher energies. Instead of using the tridiagonalization of the Hamiltonian, but in a similar spirit regarding the important target states to be kept, the spectrum can be calculated for a given $z=w+i \eta$ by using a correction vector (related to the operator $A$ that can depend on momentum $q$ ).

Following the Green's function given above, the (complex) correction vector $|x(z)\rangle$ can be defined as:

$$
|x(z)\rangle=\frac{1}{z-H} A\left|\psi_{0}\right\rangle
$$

so the Green's function can be calculated as $G(z)=\left\langle\psi_{0}\left|A^{\dagger}\right| x(z)\right\rangle$.

Separating the correction vector in real and imaginary parts $|x(z)\rangle=$ $\left|x^{r}(z)\right\rangle+i\left|x^{i}(z)\right\rangle$ we obtain

$$
\begin{aligned}
\left((H-w)^{2}+\eta^{2}\right)\left|x^{i}(z)\right\rangle & =-\eta A\left|\psi_{0}\right\rangle \\
\left|x^{r}(z)\right\rangle & =\frac{1}{\eta}(w-H)\left|x^{i}(z)\right\rangle
\end{aligned}
$$

The former equation is solved using the conjugate gradient method. In order to keep the information of the excitations at this particular energy the following states are targeted in the DMRG iterations: The ground state $\left|\psi_{0}\right\rangle$, the first Lanczos vector $A\left|\psi_{0}\right\rangle$ and the correction vector $|x(z)\rangle$. Even though only a certain energy is focused on, DMRG gives the correct excitations for an energy range surrounding this particular point so that by running several times for nearby frequencies, an approximate spectrum can be obtained for a wider region 305.

A variational formulation of the correction vector technique has been developed in 307. In order to solve Eq. 32, the following equation is minimized w.r.t $|X\rangle$ :

$$
W_{A, \eta}(\omega, X)=\left\langle X\left|(H-w)^{2}+\eta^{2}\right| X\right\rangle+\eta\left\langle\psi_{0}|A| X\right\rangle+\eta\left\langle X|A| \psi_{0}\right\rangle .
$$

For any $\eta \neq 0$ and finite $\omega$ this function has a well defined minimum for the quantum state which is solution of (32), i.e. $\left|x^{i}(z)\right\rangle$. This method is very similar to thge correction vector one, but has a smaller error in the determination of the spectral function [230]. It has been successfully applied to calculate the optical conductivity of Mott insulators 318, 319, 320, spectral functions of the 1D Hubbard insulator 316] (see Fig. 14) and away from half-filling [321] 


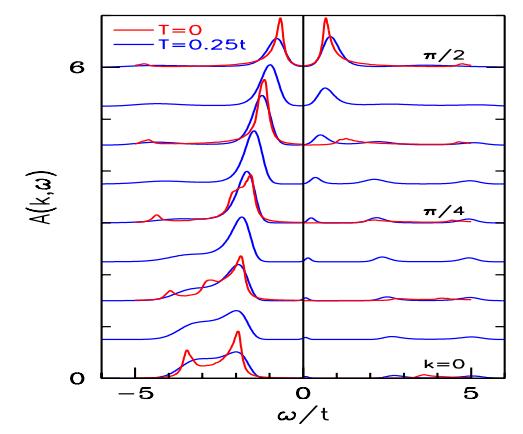

Figure 14. Single-particle spectral function for the 1D Hubbard insulator for $U=4 t$ and several momenta. The black curves are the DMRG results for $T=0$ (64 sites) and the blue curve corresponds to Quantum Monte Carlo data for $T=0.25 t$ (reprinted from Ref. 316 with permission).

and, more recently, quantum impurity spectral functions for one 322 and two impurities 323].

The correction vector model has also been applied to determine the nonlinear optical coefficients of Hubbard chains and related models 324, to calculate ac conductivity of the Bose-Hubbard model [325] and the single-impurity Anderson model. 326] It has also been recently applied to calculate the conductance through an interacting spinless fermionic systems in the linear response using the Kubo formula 327.

An interesting development for calculating response functions in single impurity systems in the presence of a magnetic field was done in 328 by using the DMRG within Wilson's NRG to obtain the Green's function. Very recently, Verstraete and collaborators proposed a variational method (by optimizing the correction vector) for evaluating Green's functions applicable to 1D quantum chain models, based on the matrix product states formalism and successfully applied to the single impurity Anderson model [286]. 


\section{Classical systems}

The DMRG has been very successfully extended to study classical systems. For a detailed description we refer the reader to Ref. 329. Since 1D quantum systems are related to 2D classical systems 330, it is natural to adapt DMRG to the classical 2D case. This method is based on the renormalization group transformation for the transfer matrix $T$ (TMRG) 331. It is a variational method that maximizes the partition function using a limited number of degrees of freedom, where the variational state is written as a product of local matrices [40]. For 2D classical systems, this algorithm is superior to the classical Monte Carlo method in accuracy, speed and in the possibility of treating much larger systems. A recent improvement of this method considering periodic boundary conditions is given in [332 and a detailed comparison between symmetric and asymmetric targeting is done in 333. TMRG has also been successfully used to renormalize stochastic transfer matrices in a study of cellular automatons 334. The calculation of thermodynamical properties of 3D classical statistical systems has been proposed [280] where the eigenstate of the transfer matrix with maximum eigenvalue is represented by the product of local tensors optimized using DMRG.

A further improvement to this method is based on the corner transfer matrix [335, the CTMRG 336, 337, 338, 339, 340,341 and can be generalized to any dimension 342 . In Ref. 343, there is an interesting analysis on the low effective theory underlying the DMRG, based on a real-space RG of the corner Hamiltonian and applied to spin chains.

It was first applied to the Ising [329, 344, 345, 346] and Potts models [347, where very accurate density profiles and critical indices were calculated. Further applications have included non-hermitian problems in equilibrium and non-equilibrium physics. In the first case, transfer matrices may be nonhermitian and several situations have been considered: a model for the Quantum Hall effect 348, the $q$-symmetric Heisenberg chain related to the conformal series of critical models 349] and the anisotropic triangular nearest and next-nearest neighbour Ising models 131. In the second case, the adaptation of the DMRG to non-equilibrium physics like the asymmetric exclusion problem [350] and reaction-diffusion problems [42,351 has shown to be very successful. It has also been applied to stochastic lattice models like in 352 and to the 2D XY model 353, to the study of simplified models for polimerized membranes in thermal equilibrium (folding of triangular lattices) 354. 


\section{Finite-temperature DMRG}

The first attempt to include the effect of finite temperature in the DMRG procedure was performed by Moukouri and Caron 355. They considered the standard DMRG taking into account several low-lying target states (see Eq. 21) to construct the density matrix, weighted with the Boltzmann factor ( $\beta$ is the inverse temperature):

$$
\rho_{i i^{\prime}}=\sum_{l} e^{-\beta E_{l}} \sum_{j} \phi_{l, i j} \phi_{l, i^{\prime} j}
$$

With this method they performed reliable calculations of the magnetic susceptibility of quantum spin chains with $S=1 / 2$ and $3 / 2$, showing excellent agreement with Bethe Ansatz exact results. They also calculated low temperature thermodynamical properties of the 1D Kondo Lattice Model [356] and of organic conductors [357. Zhang et al. 358] applied the same method in the study of a magnetic impurity embedded in a quantum spin chain.

An alternative way of incorporating temperature, called the Transfer Matrix DMRG (TDMRG) stems from the Trotter-Suzuki expansion of the partition function of a one-dimensional model, turning it into a classical two-

dimensional model, with the new axis corresponding to imaginary time (inverse temperature). The extension of the DMRG method to classical systems paved the way to study $1 \mathrm{D}$ quantum systems at non zero temperature 359, 360, 361, 341, 362 (see also a recent review in Ref. 285). In this case the system is infinite and the finiteness is in the level of the Trotter approximation. The free energy in the thermodynamic limit is determined by the largest eigenvalue of the transfer matrix. Being a high-temperature expansion, the method loses precision at low temperatures.

Very nice results have been obtained for the dimerized, $S=1 / 2$, XY model, where the specific heat was calculated involving an extremely small basis set [359] $(m=16)$, the agreement with the exact solution being much better in the case where the system has a substantial gap. It has also been used to calculate thermodynamic properties of the anisotropic $S=1 / 2$ Heisenberg model, with relative errors for the spin susceptibility of less than $10^{-3}$ down to temperatures of the order of $0.01 J$ keeping $m=80$ states [361. A complete study of thermodynamic properties like magnetization, susceptibility, specific heat and temperature dependent correlation functions for the $S=1 / 2$ and $3 / 2$ Heisenberg models was done in 363 . Other applications have been the calculation of the temperature dependence of the charge and spin gap in the Kondo insulator [364, the calculation of thermodynamic properties of ferrimagnetic chains [365] and spin ladders [136], the study of impurity properties in spin chains [366 367], frustrated quantum spin chains [368], t-J [369] and 
spin ladders [370] and dimerized frustrated Heisenberg chains 371]. Recent studies include the thermodynamics and crossover phenomena in the $1 \mathrm{D}$ t-J model using this method which led to very accurate results, as compared to the particular case of the supersymmetric limit where analytic Bethe-Ansatz results are available 372 and the calculation of the specific heat in doped anisotropic Hubbard ladders with charge-order instability 373 .

Recently, new proposals for calculating finite-temperature properties arose based on the imaginary-time evolution of the wave function in the matrixproduct [22, 375 374 ] and ancillary states [22,376] formalisms to simulate evolutions of physical quantities in real and imaginary time and at finite temperature. They are based on the so-defined projected entangled-pair states (PEPS) and the matrix product density operators (MPDO). The evolution in imaginary time to construct thermal and mixed states leads to a versatile DMRG algorithm that is restricted neither to large temperatures nor to homogeneous systems and opens the possiblity of simulating finite temperature, dissipation and decoherence effects. From the DMRG point of view, the PEPS or ancilla method is particularly appropriate. The Hilbert space is enlarged by introducing auxiliary states (in the form of an extra chain coupled to the system for example) and then the imaginary-time evolution of a pure state in the enlarged space is calculated (this process is known as "purification" in quantum information theory [26]). The ancilla sites serve as a thermodynamic bath which is traced out to obtain the thermodynamic behaviour of the original system.

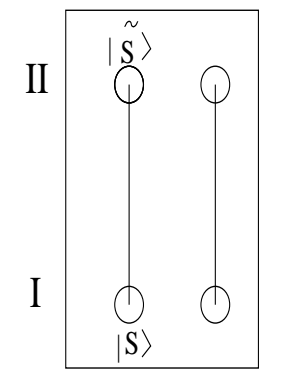

System

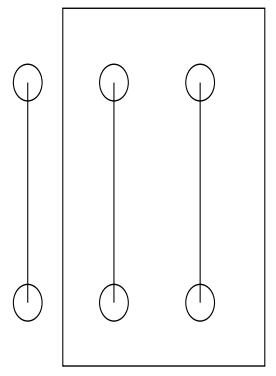

Environment

Figure 15. Schematic representation of the ancilla approach used to calculate imaginary-time evolution. The real system corresponds to the lower sites (I) and the ancillary states are labelled by II. Full lines represent maximally entangled states.

Let our original system be defined by states $\left|s_{i}\right\rangle$ and our auxiliary bath by $\left|\tilde{s}_{i}\right\rangle$ (Fig. 15). Following the notation of [376], we define the non-normalized 
pure state

$$
|\psi(\beta)\rangle=e^{-\beta H / 2}|\psi(0)\rangle
$$

where the Hamiltonian $H$ acts only on the real system (I). If $\beta=0(T \rightarrow \infty)$ this wave function is in a maximally entangled state between the original and auxiliary states:

$$
|\psi(0)\rangle=\prod_{i} \sum_{s_{i}}\left|s_{i} \tilde{s}_{i}\right\rangle
$$

The partition function for arbitrary $\beta$ is $S=\langle\psi(\beta) \mid \psi(\beta)\rangle$ and the thermal average of an operator $A$ is calculated as

$$
\langle A(\beta)\rangle=\frac{\langle\psi|A| \psi\rangle}{\langle\psi \mid \psi\rangle}
$$

To obtain the temperature-dependent wave function, one can start with the maximally entangled state $|\psi(0)\rangle$ and then evolve in imaginary time applying the Hamiltonian to the real system only. Within the DMRG framework, the blocks can be thought of as for example shown in Fig 15, where each pair $\left|s_{i} \tilde{s}_{i}\right\rangle$ forms a supersite and the maximally entangled state is represented as a full line. The imaginary-time evolution is performed with any of the real-time algorithms (for example, the adaptive algorithm [295, 290] for nearest neighbour Hamiltonians). In [376, the specific heat, magnetic susceptibility and the correlation length are calculated in spin chains with an accuracy comparable to the transfer-matrix DMRG (see Fig 16).

\subsection{Finite-temperature dynamics}

In order to include temperature in the calculation of dynamical quantities, the Transfer Matrix RG described above (TMRG) 359, 361,362 was extended to obtain imaginary time correlation functions [377,378,379]. After Fourier transformation in the imaginary time axis, analytic continuation from imaginary to real frequencies is done using maximum entropy (ME). The combination of the TMRG and ME is free from statistical errors and the negative sign problem of Monte Carlo methods but still has the extrapolation error of the analytic continuation. Since we are dealing with the transfer matrix, the thermodynamic limit (infinite system size) can be discussed directly without extrapolations. However, in the present scheme, only local quantities can be calculated.

A systematic investigation of local spectral functions is done in Ref. 379. for the anisotropic Heisenberg antiferromagnetic chain. The authors obtain 


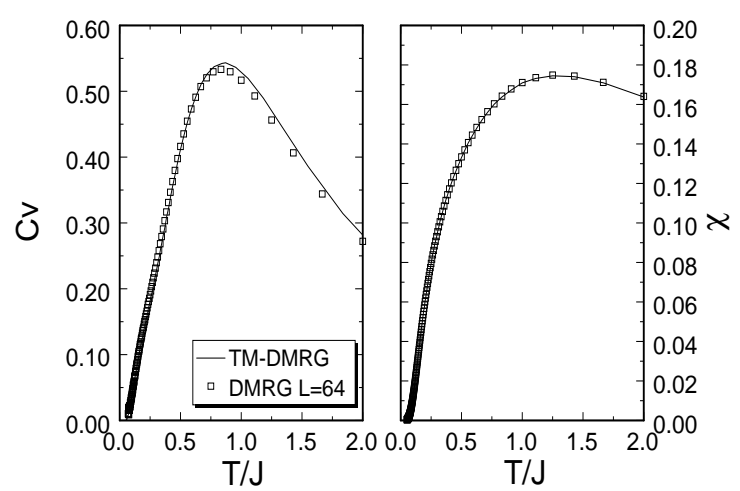

Figure 16. Specific heat and magnetic susceptibility of the Haldane chain $(S=1)$ with 64 sites calculated using the Trotter-Suzuki time-evolution algorithm. Also shown are the results using Transfer Matrix DMRG (reprinted from Ref. [376] with permission).

good qualitative results especially for high temperatures but a quantitative description of peaks and gaps are beyond the method, due to the severe intrinsic limitation of the analytic continuation. This method was also applied with great success to the 1D Kondo insulator [378 where the temperature dependence of the local density of states and local dynamic spin and charge correlation functions were calculated. A modfication of this method to avoid the use of the ill-posed analytic continuation was done in 380. by considering a path-integral approach to calculate real-time correlation functions at finite temperatures.

\section{Dynamical Mean Field Theory using DMRG}

The Dynamical Mean Field Theory (DMFT) has become one of the basic methods to calculate realistic electronic band structure in strongly correlated systems 381. At the heart of the DMFT method is the solution of an associated quantum impurity model where the environment of the impurity has to be determined self-consistently. Therefore the ability to obtain reliable DMFT solutions of lattice model Hamiltonians relies directly on the ability to solve quantum impurity models. Among the a priori exact numerical algorithms available we find the Hirsch-Fye Quantum Monte Carlo [382,383] method and Wilson's Numerical Renormalization Group (NRG) 8 8,384,385. While the former, a finite-temperature method, is very stable and accurate at the Matsubara frequencies, its main drawback is the access to real frequency quantities for the calculation of spectral functions which requires less controlled techniques for the analytic continuation of the Green functions. The second method can be 
formulated both at $T=0$ and finite (small) $T$ and provides extremely accurate results at very small frequencies, at the expense of a less accurate description of the high energy features.

In order to overcome the difficulties encountered by these other methods, we will show that DMRG can be used very reliably to solve the related impurity problem within DMFT 386. By using the DMRG to solve the impurity, no a priori approximations are made and the method provides equally reliable solutions for both gapless and gapful phases. More significantly, it provides accurate estimates for the distributions of spectral intensities of high frequency features such as the Hubbard bands, that are of main relevance for analysis of $\mathrm{X}$-ray photoemission and optical conductivity experiments.

We will now very briefly describe the method applied to the Mott transition in the Hubbard model. The Hamiltonian of the Hubbard model is defined by Eq. 15. Applying DMFT to this model leads to a mapping of the original lattice model onto an associated quantum impurity problem in a self-consistent bath. In the particular case of the Hubbard model, the associated impurity problem is the single impurity Anderson model (SIAM), where the hybridization function $\Delta(\omega)$, which in the usual SIAM is a flat density of states of the conduction electrons, is now to be determined self-consistently. More precisely, for the Hamiltonian (15) defined on a Bethe lattice of coordination $d$, one takes the limit of large $d$ and exactly maps the model onto a SIAM impurity problem with the requirement that $\Delta(\omega)=t^{2} G(\omega)$, where $G(\omega)$ is the impurity Green's function. At the self-consistent point $G(\omega)$ coincides with the local Green's function of the original lattice model [387. We take the half-bandwidth of the non interacting model as unit of energy, $t=1 / 2$.

The Green's function of the impurity problem is an important quantity in this algorithm: $\mathrm{G}_{0}(\omega)=1 /(\omega+\mu-\Delta(\omega))=1 /\left(\omega+\mu-t^{2} G(\omega)\right)$. Thus, to implement the new algorithm we shall consider [388, 389] a general representation of the hybridization function in terms of continued fractions that define a parametrization of $\Delta(\omega)$ in terms of a set of real and positive coefficients. Since it is essentially a Green's function, $\Delta(z)$ can be decomposed into "particle" and "hole" contributions as $\Delta(z)=\Delta^{>}(z)+\Delta^{<}(z)$ with $\Delta^{>}(z)=t^{2}\left\langle g s\left|c \frac{1}{z-\left(H-E_{0}\right)} c^{\dagger}\right| g s\right\rangle$ and $\Delta^{<}(z)=t^{2}\left\langle g s\left|c^{\dagger} \frac{1}{z+\left(H-E_{0}\right)} c\right| g s\right\rangle$ for a given Hamiltonian, $\mathrm{H}$ with ground-state energy $E_{0}$. By standard Lanczos technique, $\mathrm{H}$ can be tridiagonalized and the functions $\Delta^{>}(z)$ and $\Delta^{<}(z)$ can be expressed in terms of respective continued fractions [63. As first implemented in Ref. 388, 389, each continued fraction can be represented by a chain of auxiliary atomic sites whose energies and hopping amplitudes are given by the continued fraction diagonal and off-diagonal coefficients respectively.

As a result of the self-consistency condition, the two chains representing the hybridization, are "attached" to the right and left of an atomic site to obtain a new SIAM Hamiltonian, $\mathrm{H}$. In fact $\mathrm{G}_{0}(z)$ constitutes the local Green's function 
of the site plus chain system. The algorithm in Ref. 388.389, basically consists in switching on the local Coulomb interaction at the impurity site of the SIAM Hamiltonian and using the Lanczos technique to re-obtain $\Delta(z)$, iterating the procedure until the set of continued fractions coefficients converges. By using the DMRG, the number of auxiliary sites that can be used in the hybridization chains is much larger than in the exact diagonalization scheme, leading to more accurate results 390. An alternative way of using DMRG to solve the impurity problem, which does not rely on the continued fraction expansion was developed in 391.

The SIAM Hamiltonian therefore reads

$$
\begin{aligned}
H= & \sum_{\sigma, \alpha=-N_{C} ; \alpha \neq 0}^{N_{C}} a_{\alpha} c_{\alpha \sigma}^{\dagger} c_{\alpha \sigma}+\sum_{\sigma, \alpha=-\left(N_{C}-1\right) ; \alpha \neq 0,-1}^{N_{C}-1} b_{\alpha}\left(c_{\alpha \sigma}^{\dagger} c_{\alpha+1 \sigma}+h . c .\right) \\
& +\sum_{\sigma, \alpha= \pm 1} b_{0}\left(c_{\sigma}^{\dagger} c_{\alpha \sigma}+h . c .\right)+U\left(n_{\uparrow}-\frac{1}{2}\right)\left(n_{\downarrow}-\frac{1}{2}\right)
\end{aligned}
$$

with $c_{\sigma}$ being the destruction operator at the impurity site, and $c_{\alpha \sigma}$ being the destruction operator at the $\alpha$ site of the hybridization chain of $2 N_{C}$ sites. The set of parameters $\left\{a_{\alpha}, b_{\alpha}\right\}$ are directly obtained from the coefficients of the continued fraction representations of $\Delta(z)$ by the procedure just described.

In Fig 17 we show the DMFT+DMRG results (solid lines) for the density of states (DOS) for several values of increasing interaction $U$. The results are compared to the Iterated Perturbation Theory (IPT) results (dashed lines) 392, 393.

Results for the imaginary part of the Green's funtions on the Matsubara frequencies match the precise Monte Carlo solutions at low temperatures. We also obtain accurate values for the two distinct critical values of the interaction $U_{c 1}==2.39 \pm 0.02$ and $U_{c 2}=3.0 \pm 0.2$. To this end, reaching larger system sizes turned out to be important in order to perform proper extrapolations and overcome finite-size effects.

As a conclusion, using DMRG as the impurity-solver of the DMFT, increases its performance. Large systems can be considered and accurate values of the critical interactions are obtained in agreement with NRG predictions allowing for a non-trivial test of the accuracy of this method. In contrast with NRG, however, this new algorithm deals with all energy scales on equal footing which allowed us to find interesting substructure in the Hubbard bands of the correlated metallic state. The ability of the new algorithm to directly deal with the high energy scales is a very important feature which is relevant for the interpretation of high resolution photoemission spectroscopies. 381] In addition, with this method, realistic band-structure calculations of systems with a 


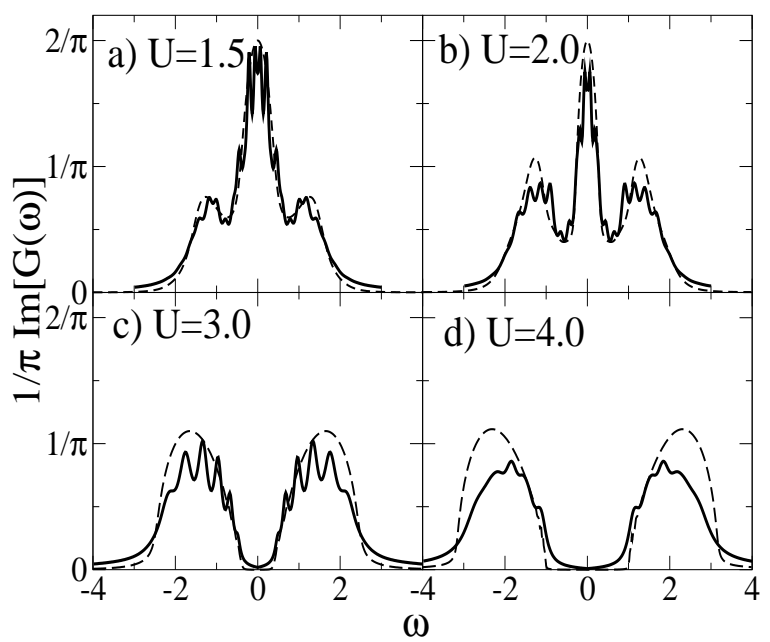

Figure 17. Density of states of the half-filled Hubbard Model. We also show the IPT results (dashed lines) (see text).

larger number of degrees of freedom and other dopings away from half-filling can be handled ??.

\section{Summary and outlook}

The DMRG method has proven to be a very reliable and versatile numerical method that can be applied to a broad spectrum of problems in physics in a variety of fields such as Condensed Matter, Statistical Mechanics, High Energy and Nuclear Physics, Quantum Chemistry and Quantum Information Theory. It is nowadays recognized as one of the most accurate and efficient numerical techniques. We have reviewed the most recent developments to DMRG among which are the very accurate real-time applications, the use of quantum information concepts which give an interesting perspective and the possibility of using it as the impurity-solver in DMFT calculations. These new improvements have triggered a great activity and numerous papers have been published applying these new techniques.

However, the DMRG is still evolving and presents very interesting potential new applications. Among the fields where DMRG has still to be exploited are the time-dependent analysis of quantum and dissipative systems, the application in DMFT band-structure calculations of more complex and realistic systems and quantum chemistry. The connection of DMRG with quantum information theory paves the way to an important new field of application and, very possibly, to dimensions higher than one. 
In view of the fact that numerical simulations constitute an essential tool in physics and quantum chemistry, a group of experts have started an interesting initiative called the ALPS (Algorithms and Libraries for Physics Simulations) project 395]. It consists of an open source of optimized software aimed at studying strongly correlated systems such as quantum magnets, strongly correlated fermionic systems and lattice bosons where methods like classical and quantum Monte Carlo, exact diagonalization and DMRG are included.

\section{Acknowledgments}

The author is grateful to numerous fruitful discussions on DMRG with most of the people involved with this method in particular with P. Horsch (who drew her attention to the method in its very early stages), S. White, I. Peschel, X. Wang, T. Xiang, T. Nishino, M. A. Martín-Delgado, G. Sierra, S. Ramasesha, E. Jeckelmann, R. Noack, U. Schollwöck, P. Schmitteckert, D. García and M. Rozenberg.

The author is Staff Researcher of the National Council for Science and Technology, CONICET (Argentina) and Fellow of the Guggenheim Foundation (2005). This project was performed under grants PICT 02 03-12742 and PICT 03-13829 of the ANPCyT (Argentina).

\section{References}

[1] S. White, Phys. Rev. Lett. 69, 2863 (1992)

[2] Density Matrix Renormalization, edited by I. Peschel, X. Wang, M. Kaulke and K. Hallberg (Series: Lecture Notes in Physics, Springer, Berlin, 1999)

[3] A. Schollwöck, The density matrix renormalization group, Rev. Mod. Phys.,77, 259, Jan. 2005 (see also R. Noack and S. Manmana, AIP Conf. Proc. 789, 93 (2005) for a more general review on numerical methods for interactin quantum systems).

[4] S. White, Phys. Rev. B 48, 10345 (1993)

[5] J. Bray and S. Chui, Phys. Rev. B 19, 4876 (1979)

[6] C. Pan and X. Chen, Phys. Rev. B 36, 8600 (1987); M. Kovarik, Phys. Rev. B 41, 6889 (1990)

[7] T. Xiang and G. Gehring, Phys. Rev. B 48, 303 (1993)

[8] K. Wilson, Rev. Mod. Phys. 47, 773 (1975)

[9] F. Verstraete et al, Phys. Rev. Lett. 94, 140601 (2005)

[10] S. White and R. Noack, Phys. Rev. Lett. 68, 3487, (1992); R. Noack and S. White, Phys. Rev. B 47, 9243 (1993)

[11] J. Gaite, Mod. Phys. Lett. A16, 1109 (2001)

[12] R. Feynman, Statistical Mechanics: A Set of Lectures, (Benjamin, Reading, MA, 1972)

[13] R. Noack and S. White in Ref. 2, Chap. 2(I)

[14] M. Martín-Delgado, G. Sierra and R. Noack, J. Phys. A: Math. Gen. 32, 6079 (1999)

[15] S. R. White, I. Affleck and D. Scalapino, Phys. Rev. B 65, 165122 (2002)

[16] See E. Dagotto, Rev. Mod. Phys. 66, 763 (1994); N. Laflorencie and D. Poilblanc, Lect. Notes Phys. 645, 227 (2004)

[17] E. R. Davidson, J. Comput. Phys. 17, 87 (1975); E.R. Davidson, Computers in Physics 7, No. 5,519 (1993).

[18] S. White, Phys. Rev. Lett. 77, 3633 (1996)

[19] T. Nishino and K. Okunishi, Phys. Soc. Jpn. 64, 4084 (1995); U. Schollwöck, Phys. Rev. B 58, 8194 (1998) and Phys. Rev. B 59, 3917 (1999) (erratum). 
[20] J. Preskill, J. Mod. Opt. 47, 127 (2000) quant-ph/9904022)

[21] T. J. Osborne and M. A. Nielsen, Quantum Intormation Processing, Volume 1, Issue 1-2, 45 (2002) (available on-line: http://www.kluweronline.com/issn/1570-0755//; ibid. Phys. Rev. A 66, 032110 (2002); F. Verstraete, M. Popp and J. I. Cirac, Phys. Rev. Lett. 92, 027901 (2004)

[22] F. Verstraete and J. Cirac, preprint, cond-mat/0407066

[23] F.Verstraete, D. Porras and C. Cirac, Phys. Rev. Lett. 93227205 (2004); F. Verstraete, M. A. Martín-Delgado and J. I. Cirac, Phys. Rev. Lett. 92, 087201 (2004)

[24] J. Latorre, E. Rico and G. Vidal, Quant. Inf. Comp. 6, 48 (2004) (quant-ph/0304098); J. Gaite, Proceedings of the conference TH2002, quant-ph/0301120

[25] A. Galindo and M. A. Martín-Delgado, Rev. Mod. Phys. 74, 347 (2002)

[26] E. Schmidt, Math. Ann. 63, 433 (1906); M. Nielsen and I. L. Chuang, Quantum computation and quantum information (Cambridge University Press), Cambridge (2000)

[27] C. H. Bennet, G. Brassard, S. Popescu and B. Schumacher, Phys. Rev. Lett. 76, 722 (1996)

[28] Ö. Legeza and J. Sólyom, Phys. Rev. B 68, 195116 (2003)

[29] C. Callan and F. Wilczek, Phys. Lett. B 333, 55 (1994); J. Gaite, Mod. Phys. Lett. A 16, 1109 (2001)

[30] G. Vidal, J. Latorre, E. Rico and A. Kitaev, Phys. Rev. Lett. 90, 227902 (2003)

[31] V. Korepin, Phys. Rev. Lett. 92, 96402 (2004)

[32] N. Laflorencie, E. Sorensen, M.-S. Chang and I. Affleck, Phys. Rev. Lett. 96, 100603 (2006)

[33] G. De Chiara, S. Montenegro, P. Calabrese and R. Fazio, preprint, cond-mat/0512586 J. Stat. Mech., P03001 (2006)

[34] J. Zhao, I. Peschel and X. Wang, Phys. Rev. B 73024417 (2006)

[35] Ö. Legeza and J. Sólyom, preprint, cond-mat/0511081 Ö. Legeza, K. Buchta and J. Sólyom, preprint, cond-mat/0511082

[36] J. Rissler, R. Noack and S. White, cond-mat/0508524 to appear in Chem. Phys. (2006)

[37] P. Zanardi, Phys. Rev. A 65, 42101 (2002); S.-J. Gu, S.-S. Deng, Y.-Q. Lin and H.-Q. Lin, Phys. Rev. Lett. 93, 86402 (2004)

[38] S.-S. Deng, S.-J. Gu and H.-Q. Lin, preprint, cond-mat/0511103

[39] I. Affleck, T. Kennedy, E. Lieb and H. Tasaki, Phys. Rev. Lett. 59, 799 (1987)

[40] S. Östlund and S. Rommer, Phys. Rev. Lett. 75, 3537 (1995); S. Rommer and S. Östlund, Phys. Rev. B 55, 2164 (1997); M. Andersson, M. Boman and S. Östlund, Phys. Rev. B 59, 10493 (1999); H. Takasaki, T. Hikihara and T. Nishino, J. Phys. Soc. Jpn. 68, 1537 (1999)

[41] J. Dukelsky et al, Europhys. Lett. 43, 457 (1997)

[42] I. Peschel and M. Kaulke, in Ref. 2], Chap. 3.1(II)

[43] K. Okunishi, Y. Hieida and Y.Akutsu, Phys. Rev. E 59, R6227 (1999)

[44] I. Peschel, M. Kaulke and Ö. Legeza, Annalen der Physik 8, 153 (1999), cond-mat/9810174)

[45] Ö. Legeza and G. Fáth, Phys. Rev. B 53, 14349 (1996); M-B Lepetit and G. Pastor, Phys. Rev. B 58, $12691(1998)$

[46] M. C. Chung and I. Peschel, Phys. Rev. B 64, 064412 (2001)

[47] C. Degli Esposti Boschi and F. Ortolani, Eur. Phys. J. B 41, 503 (2004)

[48] F. Verstraete and J. I. Cirac, Phys. Rev. B 73, 094423 (2006)

[49] M. C. Chung and I. Peschel, Phys. Rev. B 62, 4191 (2000)

[50] Ö. Legeza and J. Sólyom, Phys. Rev. B 70, 205118 (2004)

[51] S. White, preprint, Phys. Rev. B 72, 180403 (2005)

[52] M. A. Martín-Delgado and G. Sierra, Int. J. Mod. Phys. A, 11, 3145 (1996)

[53] G. Sierra and M. A. Martín-Delgado, in "The Exact Renormalization Group " by a Krasnitz, R Potting, Y A Kubyshin and P. S. de Sa (Eds.) World Scientific Pub Co; ISBN: 9810239394, (1999), (cond-mat/9811170)

[54] I. Peschel, J. Phys. A: Math. Gen. 36, L205 (2003)

[55] G. Sierra and T. Nishino, Nucl. Phys. B 495, 505 (1997) (cond-mat/9610221)

[56] W. Tatsuaki, Phys. Rev. E 61, 3199 (2000); T. Wada and T. Nishino, cond-mat/0103508 (Proceedings of the Conference on Computational Physics 2000 (CCP2000), Gold Coast, Queensland, Australia, 3-8 December 2000)

[57] I. P. McCulloch, M. Gulacsi, S. Caprara and A. Juozapavicius, J. Low Temp. Phys. 117, 323 (1999)

[58] E. S. Sørensen and I. Affleck, Phys. Rev. B 51, 16115 (1995)

[59] I.P. McCulloch and M. Gulacsi, Europhys. Lett. 57, 852 (2002); I. P. McCulloch, A. R. Bishop and M. Gulacsi, Philos. Mag. B 81, 1603 (2001)

[60] S. Ramasesha, S. Pati, H. R. Krishnamurthy, Z. Shuai and J. L. Brédas, Phys. Rev. B 54, 7598 
(1996); S. Ramasesha, S. Pati, H. R. Krishnamurthy, Z. Shuai and J. L. Brédas, Synth. Metals 85, 1019 (1997)

[61] M.S.L. du Croo de Jongh and J.M.J. van Leeuwen, Phys. Rev. B 57, 8494 (1998)

[62] H-H. Lin et al, preprint, cond-mat/0410654

[63] K. Hallberg, Phys. Rev. B 52, 9827 (1995).

[64] S. R. White and D. Huse, Phys. Rev. B 48, 3844 (1993)

[65] E. S. Sørensen and I. Affleck, Phys. Rev. B 49, 13235 (1994)

[66] E. S. Sørensen and I. Affleck, Phys. Rev. B 49, 15771 (1994); E. Polizzi, F. Mila and E. Sørensen, Phys. Rev. B 58, 2407 (1998)

[67] C. Batista, K. Hallberg and A. Aligia, Phys. Rev. B 58, 9248 (1998)

[68] C. Batista, K. Hallberg and A. Aligia, Phys. Rev. B 60, 12553 (1999)

[69] K. Hallberg, C. Batista and A. Aligia, Physica B, 259, 1017 (1999)

[70] E. Jannod, C. Payen, K. Schoumacker, C. Batista, K. Hallberg and A. Aligia, Phys. Rev B, 62, $2998(2000)$

[71] M. Laukamp et al., Phys. Rev. B 5, 10755 (1998)

[72] T-K. Ng, J. Lou and Z. Su, Phys. Rev. B 61, 11487 (2000)

[73] J. Lou, S. Qin, T-K. Ng, Z. Su and I. Affleck, Phys. Rev. B 62, 3786 (2000)

[74] S-W Tsai and J. B. Marston, Ann. Phys. (Leipzig) 8, Special Issue, 261 (1999)

[75] M. Sieling, U. Löw, B. Wolf, S. Schmidt, S. Zvyagin and B.Lüthi, Phys. Rev. B 61, 88 (2000)

[76] Y. Nishiyama, K. Totsuka, N. Hatano and M. Suzuki, J. Phys. Soc. Jpn 64, 414 (1995)

[77] S. Qin, T. Ng and Z-B Su, Phys. Rev. B 52, 12844 (1995)

[78] U. Schollwöck and T. Jolicoeur, Europhys. Lett. 30, 493 (1995)

[79] X. Wang, S. Qin and Lu Yu, Phys. Rev. B. 60, 14529 (1999)

[80] M. Capone and S. Caprara, Phys. Rev. B 64, 184418 (2001)

[81] J. Lou, X. Dai, S. Qin, Z. Su and L. Yu, Phys. Rev. B 60, 52 (1999)

[82] E. Ercolessi, G. Morandi, P. Pieri and M. Roncaglia, Europhys. Lett. 49, 434 (2000)

[83] S. Qin, X. Wang and Lu Yu, Phys. Rev. B 56, R14251 (1997)

[84] M. Rizzi et al, Phys. Rev. Lett. 95, 240404 (2005)

[85] K. Hallberg, P. Horsch and G. Martínez, Phys. Rev. B, 52, R719 (1995)

[86] H. E. Boos, V. E. Korepin, Y. Nishiyama and M. Shiroishi, J. Phys. A35, 4443 (2002); V. E. Korepin, S. Lukyanov, Y. Nishiyama and M. Shiroishi, Phys. Lett. A 312, 21 (2003)

[87] M. Shiroishi, M. Takahashi and Y. Nishiyama, J. Phys. Soc. Jpn. 70, 3535 (2001)

[88] T. Hikihara and A. Furusaki, Phys. Rev. B 58, R583 (1998)

[89] G. Fath, Phys. Rev. B 68, 134445 (2003)

[90] J. Lou, S. Qin, C. Chen, Z. Su and L. Yu, Phys. Rev. B 65, 064420 (2002)

[91] J. Lou, S. Qin and C. Chen, Phys. Rev. Lett. 91, 087204 (2003)

[92] F. Capraro and C. Gros, Eur. Phys. J. B 29, 35 (2002)

[93] Y. Hieida, K. Okunishi and Y. Akutsu, Phys. Rev. B 64, 224422 (2001)

[94] T. Byrnes, R. Bursill, H-P. Eckle, C. Hamer and A. Sandvik, Phys. Rev. B66, 195313 (2002)

[95] S-W. Tsai and J. B. Marston, Phys. Rev. B 62, 5546 (2000)

[96] M-B. Lepetit, M. Cousy and G. Pastor, Eur. Phys. J. B 13, 421 (2000); H. Otsuka, Phys. Rev. B 53, $14004(1996)$

[97] W. Zhuo, X. Wang and Y. Wang, preprint, cond-mat/0501693

[98] K. Hallberg, X. Wang, P. Horsch and A. Moreo, Phys. Rev. Lett. 76, 4955 (1996)

[99] A. Moreo, Phys. Rev. B 35, 8562 (1987); T. Ziman and H. Schulz, Phys. Rev. Lett 59, 140 (1987)

[100] Y. Yamashita, N. Shibata and K. Ueda, Phys. Rev. B 58, 9114 (1998); Y. Yamashita, N. Shibata and K. Ueda, J. Phys. Soc. Jpn. 69, 242 (2000); Y. Yamashita, N. Shibata and K. Ueda, Phys. Rev. B 61, 4012 (2000)

[101] A. Onufriev and B. Marston, Phys. Rev. B 59, 12573 (1999)

[102] C. Itoi, S. Qin and I. Affleck, Phys. Rev. B 61, 6747 (2000)

[103] W. Yu and S. Haas, Phys. Rev. B 63, 024423 (2001)

[104] R. J. Bursill, T. Xiang and G. A. Gehring, J. Phys. A 282109 (1994)

[105] R. J. Bursill, G. A. Gehring, D. J. J. Farnell, J. B. Parkinson, T. Xiang and C. Zeng, J. Phys. C 78605 (1995)

[106] U. Schollwöck, Th. Jolicoeur and T. Garel, Phys. Rev. B 53, 3304 (1996)

[107] R. Chitra, S. Pati, H. R. Krishnamurthy, D. Sen and S. Ramasesha, Phys. Rev. B 52, 6581 (1995); S. Pati, R. Chitra, D. Sen, H. R. Krishnamurthy and S. Ramasesha, Europhys. Lett. 33, 707 (1996); J. Malek, S. Drechsler, G. Paasch and K. Hallberg, Phys. Rev. B 56, R8467 (1997); E. Sørensen et al in Ref. 2, Chap.1.2 (Part II) and references therein; D. Augier, E. Sørensen, 
J. Riera and D. Poilblanc, Phys. Rev. B 60, 1075 (1999)

[108] Y. Kato and A. Tanaka, J. Phys. Soc. Jpn. 63, 1277 (1994)

[109] S. R. White and I. Affleck, Phys. Rev. B 54, 9862 (1996)

[110] G. Bouzerar, A. Kampf and G. Japaridze, Phys. Rev. B 58, 3117 (1998); G. Bouzerar, A. Kampf and F. Schönfeld, cond-mat/9701176 unpublished; M-B. Lepetit and G. Pastor, Phys. Rev. B 56, 4447 (1997)

[111] M. Kaburagi, H. Kawamura and T. Hikihara, J. Phys. Soc. Jpn. 68. 3185 (1999)

[112] T. Hikihara, J. Phys. Soc. Jpn. 71, 319 (2002); T. Hikihara, M. Kaburagi, H. Kawamura and T. Tonegawa, Phys. Rev. B 63, 174430 (2001)

[113] S. Pati, S. Ramasesha and D. Sen, Phys. Rev. B 55, 8894 (1996); J. Phys. Cond. Matt. 9, 8707 (1997); T. Tonegawa, T. Hikihara, T. Nishino, M. Kaburagi, S. Miyashita, H.J. Mikeska, J. Mag. 177-181, 647 (1998)

[114] M. Azzouz, L. Chen and S. Moukouri, Phys. Rev. B 506223 (1994); S. R. White, R. M. Noack and D. J. Scalapino, Phys. Rev. Lett. 73886 (1994); K. Hida, J. Phys. Soc. Jpn. 644896 (1995); T. Narushima, T. Nakamura and S. Takada, J. Phys. Soc. Jpn. 644322 (1995); U. Schollwöck and D. Ko, Phys. Rev. B 53240 (1996); G. Sierra, M. A. Martín-Delgado, S. White and J. Dukelsky, Phys. Rev. B 59, 7973 (1999); S. White, Phys. Rev. B 53, 52 (1996)

[115] A. Kawaguchi, A. Koga, K. Okunishi and N. Kawakami, Phys. Rev. B 65, 214405 (2002)

[116] A. Trumper and C. Gazza, Phys. Rev. B 64, 134408 (2001)

[117] M. Roger, Phys. Rev. B 63, 144433 (2001)

[118] T. Hikihara and A. Furusaki, Phys. Rev. B 63, 134438 (2001)

[119] L. Capriotti, D. Scalapino and S. White, Phys. Rev. Lett. 93, 177004 (2004)

[120] M. A. Martín-Delgado, J. Rodriguez-Laguna and G. Sierra, Phys. Rev. B 72104435 (2005)

[121] A. Laeuchli, G. Schmid and M. Troyer, Phys. Rev. B 67, 100409 (2003)

[122] T. Hikihara, T. Momoi and X. Hu, Phys. Rev. Lett. 90, 087204 (2003)

[123] T. Nunner, P. Brune, T. Kopp, M. Windt and M. Grüninger, Phys. Rev. B 66, 180404 (2002)

[124] Y. Honda and T. Horiguchi, preprint, cond-mat/0106426

[125] S. K. Pati and R. R. P. Singh, Phys. Rev. B 60, 7695 (1999); S. R. White and R. Singh, Phys. Rev. Lett. 85, 3330 (2000)

[126] N. Maeshima, M. Hagiwara, Y. Narumi, K. Kindo, T. C. Kobayashi and K. Okunishi,Journal of Physics: Cond. Matt. 15, 3607 (2003); N. Maeshima and K. Okunishi, Phys. Rev. B 62, 934-939 (2000)

[127] C. Itoi and S. Qin, Phys. Rev. B 63, 224423 (2001)

[128] J. Lou, J. Dai, S. Qin, Z. Su and L. Yu, Phys. Rev. B 62, 8600 (2000)

[129] C. Raghu, I. Rudra, S. Ramasesha and D. Sen, Phys. Rev. B 62, 9484 (2000)

[130] A. Honecker, M. Kaulke and K.D. Schotte, Eur. Phys. J. B 15, 423 (2000)

[131] A. Gendiar and A. Surda, Phys. Rev. B 62, 3960 (2000)

[132] H. Otsuka, Y. Okabe an K. Okunishi, Phys. Rev. E 73 035105(R) (2006)

[133] J.-B. Fouet et al, Phys. Rev. B 73, 014409 (2006)

[134] K. Tandon, S. Lal, S. K. Pati, S. Ramasesha and D. Sen, Phys. Rev. B 59, 396 (1999); R. Citro, E. Orignac, N. Andrei, C. Itoi and S. Qin, J. Phys.: Cond. Mat. 12, 3041 (2000)

[135] J. Lou, C. Chen and S. Qin, Phys. Rev. B 64, 144403 (2001)

[136] X. Wang and L. Yu, Phys. Rev. Lett. 84, 5399 (2000)

[137] J. Silva-Valencia and E. Miranda, Phys. Rev. B 65, 024443 (2002); J. Silva-Valencia, J. C. Xavier and E. Miranda, Phys. Rev. B 71, 024405 (2005)

[138] B. Gu, G. Su and S. Gao, J. Phys: Cond. Matt.17, 6081 (2005)

[139] S. K. Pati, S. Ramasesha and D. Sen, in Magnetism: Molecules to Materials IV, eds. J.S. Miller and M. Drillon (Wiley-VCH Weinheim, 2002), Chapter 4, (cond-mat/0106621)

[140] L. Chen and S. Moukouri, Phys. Rev. B 531866 (1996); S. J. Qin, S. D. Liang, Z. B. Su and L. Yu, Phys. Rev. B 52 R5475 (1995); R. Noack in Ref. [2], Chap.1.3 (Part II); S. Daul and R. Noack, Phys. Rev. B 61, 12361 (2000); M. Vojta, R. Hetzel and R. Noack, Phys. Rev. B 60, R8417 (1999)

[141] K. Penc, K. Hallberg, F. Mila and H. Shiba, Phys. Rev. Lett. 77, 1390 (1996); Phys. Rev. B, $\mathbf{5 5}, 15475(1997)$

[142] N. Bulut, Advances in Physics 51, 1587 (2002)

[143] A. Malvezzi, T. Paiva and R. dos Santos, Phys. Rev. B 66, 064430 (2002)

[144] E. Jeckelmann, Phys. Rev. Lett. 89, 236401 (2002); G. P. Zhang, Phys. Rev. B 68, 153101 (2003) and erratum Phys. Rev. B 69, 199902(E) (2004); see also comment on this paper by E. Jeckelmann, Phys. Rev. B 71, 197101 (2005) and the reply by G. P. Zhang, Phys. Rev. B 71, 197102 (2005). 
[145] Y. Z. Zhang, C. Wu and H. Q.Lin, Phys. Rev. B 65, 115101 (2002); Y. Z. Zhang, C. Q. Wu and H. Q. Lin, Phys. Rev. B 72, 125126 (2005); Y. Z. Zhang, Phys. Rev. Lett. 92, 246404 (2004)

[146] A. P. Kampf, M. Sekania, G. I. Japaridze, Ph. Brune, J. Phys.: Condens. Matter 155895 (2003)

[147] C. Aebischer, D. Baeriswyl and R. M. Noack, Phys. Rev. Lett. 86, 468 (2001)

[148] R. Arita and H. Aoki, Phys. Rev. B 61, 12261 (2000); R. Arita, Y. Shimoi, K. Kuroki and H. Aoki, Phys. Rev. B 57, 10609 (1998)

[149] S. Daul, Eur. J. Phys. B 14, 649 (2000)

[150] S. Qin, J. Lou, T. Xiang, G-S. Tian and Z. Su, Phys. Rev. B 68, 045110 (2003)

[151] A. Aligia, K. Hallberg, C. Batista and G. Ortiz, Phys. Rev. B 61, 7883 (2000)

[152] A. Weisse, R. Bursill, C. Hamer and Z. Weihong, preprint, cond-mat/0511528

[153] V. Meden, W. Metzner, U. Schollwöck, O. Schneider, T. Stauber and K. Schoenhammer, , Europhys. J B 16, 631 (2000)

[154] P. Farkasovsky, Phys. Rev. B 65, 081102 (2002)

[155] C. Schuster, R. Roemer and M. Schreiber, Phys. Rev. B 65, 115114 (2002)

[156] K. Hida, Phys. Rev. Lett. 86, 1331 (2001)

[157] S. Ejima, F. Gebhard and S. Nishimoto, Europhys. Lett. 70, 492 (2005)

[158] W. Lay and J. Rudnick, Phys. Rev. Lett. 88, 057203 (2002); Y. Nishiyama, J. Phys. A34, 11215 (2001); S. G. Chung, Phys. Rev. E 62, 3262 (2000)

[159] B. Srinivasan and M. B. Lepetit, Phys. Rev. B 66, 024421 (2002); H. Sakamoto, T. Momoi and K. Kubo, Phys. Rev. B 65, 224403 (2002)

[160] J. Xavier, H. Onishi, T. Hotta and E. Dagotto, Phys. Rev. B 73, 014405 (2006)

[161] L. Hozoi and S. Nishimoto, preprint, cond-mat/0512219

[162] K. Hamacher, C. Gros and W. Wenzel, Phys. Rev. Lett. 88, 217203 (2002)

[163] M. Vojta, A. Huebsch and R. M. Noack, Phys. Rev. B 63, 045105 (2001); Z. Weihong, J. Oitmaa, C.J. Hamer and R.J. Bursill, J. Phys. C 13, 433 (2001)

[164] Youngho Park, S. Liang and T. K. Lee, Phys. Rev. B 59, 2587 (1999)

[165] Y. Yan, S. Mazumdar and S. Ramasesha, preprint, cond-mat/0601481

[166] B. Edegger, H. Evertz and R. Noack, preprint, cond-mat/0510325

[167] S. Rommer, S. R. White and D. J. Scalapino, Phys. Rev. B 61, 13424 (2000)

[168] G. Hager et al, preprint, Phys. Rev. B 71, 075108 (2005)

[169] J. O. Fjaerestad, J. B. Marston and U. Schollwöck , Ann. Phys. (NY) 321894 (2006), cond-mat/0412709

[170] B. Edegger, H. G. Evertz and R. M. Noack, Phys. Rev. B 72, 085131 (2005)

[171] G. Roux et al., Phys. Rev. B 72, 014523 (2005)

[172] R. M. Noack, S. R. White and D. J. Scalapino, Phys. Rev. Lett. 73, 882 (1994); S. R. White, R. M. Noack and D. J. Scalapino, J. Low Temp. Phys. 99, 593 (1995); R. M. Noack, S. R. White and D. J. Scalapino, Europhys. Lett. 30, 163 (1995); C. A. Hayward, D. Poilblanc, R. M. Noack, D. J. Scalapino and W. Hanke, Phys. Rev. Lett. 75, 926 (1995); S. White and D. Scalapino, Phys. Rev. Lett. 81, 3227 (1998); E. Jeckelmann, D. Scalapino and S. White, Phys. Rev. B 58, $9492(1998)$

[173] S. Nishimoto, E. Jeckelmann and D. Scalapino, Phys. Rev. B 66, 245109 (2002)

[174] J. B. Marston, J. O. Fjaerestad and A. Sudbo, Phys. Rev. Lett. 89, 056404 (2002)

[175] U. Schollwöck, S. Chakravarty, J. O. Fjaerestad, J. B. Marston and M. Troyer, Phys. Rev. Lett. 90, $186401(2003)$

[176] L. G. Caron and C. Bourbonnais, Phys. Rev. B 66, 045101 (2002)

[177] G. Fano, F. Ortolani, A. Parola and L. Ziosi, Phys. Rev. B 60, 15654 (1999)

[178] N. Shibata and D. Yoshioka, Phys. Rev. Lett. 86, 5755 (2001)

[179] N. Shibata and D. Yoshioka, Physica E 12, 43 (2002)

[180] N. Shibata and D. Yoshioka, J. Phys. Soc. Jpn. 75, 043712 (2006)

[181] V. Meden and U. Schollwöck, Phys. Rev. B 67, 035106 (2003); F. Carvalho Dias, I. Pimentel and M. Henkel, Phys. Rev. B 73, 075109 (2006)

[182] T. A. Costi, P. Schmitteckert, J. Kroha and P. Wölfle, Phys. Rev. Lett. 73, 1275 (1994); S. Eggert and I. Affleck, Phys. Rev. Lett. 75, 934 (1995); E. S. Sørensen and I. Affleck, Phys. Rev. B 51. 16115 (1995); X. Q. Wang and S. Mallwitz, Phys. Rev. B 53, R492 (1996); W. Wang, S. J. Qin, Z. Y. Lu, L. Yu and Z. B. Su, Phys. Rev. B 53, 40 (1996); C. C. Yu and M. Guerrero, Phys. Rev. B 54, 15917 (1996); A. Furusaki and T. Hikihara, Phys. Rev. B 58, 5529 (1998)

[183] K. Hallberg and R. Egger, Phys. Rev. B 55, 8646 (1997)

[184] C. Schuster and U. Eckern, Ann. Phys. (Leipzig) 11, 901-915 (2002); W. Zhang, J. Igarashi and P. Fulde, Phys. Rev. B 56, 654 (1997)

[185] V. Meden, W. Metzner, U. Schollwöck and K. Schoenhammer, Phys. Rev. B 65, 045318 (2002) 
[186] S. Andergassen et al, Phys. Rev. B 73, 045125 (2006)

[187] C. C. Yu and S. R. White, Phys. Rev. Lett. 71, 3866 (1993); C. C. Yu and S. R. White, Physica B 199, 454 (1994)

[188] S. Moukouri and L. G. Caron, Phys. Rev. B 52, 15723 (1995); N. Shibata, T. Nishino, K. Ueda and C. Ishii, Phys. Rev. B 53, R8828 (1996); M. Guerrero and R. M. Noack, Phys. Rev. B 53, $3707(1996)$

[189] H. Otsuka and T. Nishino, Phys. Rev. B 52, 15066 (1995); S. Moukouri, L. G. Caron, C. Bourbonnais and L. Hubert, Phys. Rev. B 51, 15920 (1995)

[190] J. C. Xavier, E. Novais and E. Miranda, Phys. Rev. B 65, 214406 (2002)

[191] T. Yamamoto, R. Manago and Y. Mori, J. Phys. Soc. Jpn. 72, 3204 (2003)

[192] I. P. McCulloch, A. Juozapavicius, A. Rosengren and M. Gulacsi, Phys. Rev. B 65, 052410 (2002)

[193] M. Guerrero and R.M. Noack, Phys. Rev. B 63, 144423 (2001)

[194] S. Watanabe, J. Phys. Soc. Jpn. 69, 2947 (2000)

[195] J. Xavier, R. Pereira, E. Miranda and I. Affleck, Phys. Rev. Lett. 90, 247204 (2003)

[196] S. Watanabe, M. Imada and K. Miyake, J. Phys. Soc. Jpn. 75043710 (2006)

[197] S. Watanabe, Y. Kuramoto, T. Nishino and N. Shibata, J. Phys. Soc. Jpn 68, 159 (1999)

[198] J. Moreno, S. Qin, P. Coleman and L. Yu, Phys. Rev. B 64, 085116 (2001)

[199] T. Schauerte et al, Phys. Rev. Lett. 94, 147201 (2005)

[200] S. Moukouri, L. Chen and L. G. Caron, Phys. Rev. B 53, R488 (1996)

[201] J. Riera, K. Hallberg and E. Dagotto, Phys. Rev. Lett. 79, 713 (1997); E. Dagotto et al., Phys. Rev. B 58, 6414 (1998)

[202] D. García, K. Hallberg, C. Batista, M. Avignon and B. Alascio, Phys. Rev Lett., 85, 3720 (2000); D. J. García, K. Hallberg, C. D. Batista, S. Capponi, D. Poilblanc, M. Avignon and B. Alascio, Phys. Rev. B. 65, 134444 (2002)

[203] B. Ammon and M. Imada, J. Phys. Soc. Jpn. 70, 547 (2001)

[204] D. Neuber et al, Phys. Rev. B 73, 014401 (2006)

[205] D. García, K. Hallberg, B. Alascio and M. Avignon, Physical Rev. Lett., 93, 17, 177204 (2004)

[206] I.P. McCulloch, A. Juozapavicius, A. Rosengren and M. Gulacsi, Phys. Rev. B, 65, 52410 (2002)

[207] K. Hida, J. Phys. Soc. Jpn. 65, 895 (1996) and 3412 (1996) (erratum); J. Phys. Soc. Jpn. 66, 330 (1997); J. Phys. Soc. Jpn. 66, 3237 (1997); Prog. Theor. Phys. Suppl. 145, 320 (2002); K. Hida, preprint, cond-mat/0602016

[208] P. Lajkó, E. Carlon, H. Rieger and F. Iglói, Phys. Rev. B 72, 094205 (2005)

[209] K. Hida, J. Phys. Soc. Jpn. 68, 3177 (1999)

[210] K. Hida, Phys. Rev. Lett. 83, 3297 (1999)

[211] F. Igloi, R. Juhasz and P. Lajko, Phys. Rev. Lett. 86, 1343 (2001)

[212] R. Mélin, Y-C. Lin, P. Lajkó, H. Rieger and F. Iglói, Phys. Rev. B 65, 104415 (2002)

[213] P. Schmitteckert, T. Schulze, C. Schuster, P. Schwab and U. Eckern, Phys. Rev. Lett. 80, 560 (1998); P. Schmitteckert and U. Eckern, Phys. Rev. B 53, 15397 (1996)

[214] D. Weinmann, P. Schmitteckert, R. Jalabert and J. Pichard, Eur. Phys. J. B 19, 139-156 (2001)

[215] P. Schmitteckert, R. Jalabert, D. Weinmann and J. L. Pichard, Phys. Rev. Lett. 81, 2308 (1998); E. Gambetti, Phys. Rev. B 72, 165338 (2005)

[216] E. Carlon, P. Lajko and F. Iglói, Phys. Rev. Lett. 87, 277201 (2001)

[217] I. Maruyama, N. Shibata and K. Ueda, J. Phys. Soc. Jpn. 73, 3239 (2004); ibid J. Phys. Soc. Jpn. 73, 434 (2004)

[218] M. Sade et al., Phys. Rev. B 71, 153301 (2005)

[219] R. Berkovits, F. von Oppen and Y. Gefen, Phys. Rev. Lett. 94, 076802 (2005)

[220] F. Ye etal, Phys. Rev. B 72, 233409 (2005)

[221] A. L. Chernyshev, A. H. Castro Neto and S. White, Phys. Rev. Lett. 94, 036407 (2005)

[222] , B. Paredes et al, Nature 429, 277 (2004); T. Kinoshita, T. Wenger and D. S. Weiss, Science 305, 1125 (2004)

[223] B. Schmidt, L. Plimak and M. Fleischhauer, Phys. Rev. A 71, 041601 (2005)

[224] X.-L Deng, D. Porras and J. Cirac, Phys. Rev. B 72, 075351 (2005)

[225] T. Oka and N. Nagaosa, Phys. Rev. Lett. 95, 266409 (2005)

[226] L. Caron and S. Moukouri, Phys. Rev. Lett. 76, 4050 (1996); L. Caron and S. Moukouri, Phys. Rev. B 56, R8471 (1997)

[227] E. Jeckelmann and S. White, Phys. Rev. B 57, 6376 (1998)

[228] R. Noack, S. White and D. Scalapino in Computer Simulations in Condensed Matter Physics VII, edited by D. Landau, K.-K. Mon and H.-B. Schüttler (Springer Verlag, Heidelberg and 
Berlin, 1994)

[229] E. Jeckelmann, C. Zhang and S. White in Ref. 2], Chap. 5.1 (II)

[230] E. Jeckelmann and H. Fehske, preprint, Proc. of the "Enrico Fermi" Int. School of Physics, Varenna, Italy, June 2005 cond-mat/0510637

[231] M. Tezuka, R. Arita and H. Aoki, Phys. Rev. Lett. 95, 226401 (2005); ibid. Physica B 359-361, $708(2005)$

[232] C. Zhang, E. Jeckelmann and S. White, Phys. Rev. Lett. 80, 2661 (1998); E. Jeckelmann, C. Zhang and S. R. White, Physical Review B 60, 7950 (1999)

[233] H. Matsueda, T. Tohyama and S. Maekawa, preprint, cond-mat/0511068

[234] Y. Nishiyama, Phys. Rev. B 64, 064510 (2001)

[235] Y. Nishiyama, Eur. Phys. J. B 12, 547 (1999)

[236] W. Barford and R. J. Bursill, Phys. Rev. Lett. 95, 137207 (2005)

[237] R. Bursill, Y. McKenzie and C. Hammer, Phys. Rev. Lett. 80, 5607 (1998); R. Bursill, Y. McKenzie and C. Hammer, Phys. Rev. Lett. 83, 408 (1999); R. Bursill, Phys. Rev. B 60, 1643 (1999)

[238] R. Pai, R. Pandit, H. Krishnamurthy and S. Ramasesha, Phys. Rev. Lett. 76, 2937 (1996) (see also the comment by N. V. Prokof'ev and B. V. Svistunov, Phys. Rev. Lett. 80, 4355 (1998)); S. Rapsch, U. Schollwöck and W. Zwerger, Europhys. Lett. 46, 559 (1999)

[239] T. Kühner and H. Monien, Phys. Rev. B 58, R14741 (1998)

[240] I. Peschel and M-C. Chung, J. Phys. A: Math. Gen. 32, 8419 (1999)

[241] P. Maurel and M-B. Lepetit, Phys. Rev. B 62, 10744 (2000); P. Maurel, M-B. Lepetit and D. Poilblanc, Eur. Phys. J. B 21, 481 (2001)

[242] T. Xiang, Phys. Rev. B 53, R10445 (1996)

[243] S. Nishimoto, E. Jeckelmann, F. Gebhard and R. Noack, Phys. Rev. B 65, 165114 (2002)

[244] D. Porras, F. Verstraete and J. I. Cirac, Phys. Rev. B 73, 014410 (2006)

[245] J. Dukelsky and G. Sierra, Phys. Rev. Lett. 83, 172 (1999); ibid. Phys. Rev. B 61, 12302 (2000); J. Dukelsky and S. Pittel, Phys. Rev. C 63, R061303 (2001)

[246] J. Dukelsky and S. Pittel, Rep. Prog. Phys. 67, 513 (2004); S. Pittel et al., Rev. Mex. Fis. 49S4, 82 (2003)

[247] D. Gobert, M. Schechter, U. Schollwöck and J. Von Delft, Phys. Rev. Lett. 93, 186402 (2004); D. Gobert, U. Schollwöck and J. Von Delft, Eur. Phys. J. B 38, 501 (2004)

[248] J. Dukelsky and G. Dussel, Phys. Rev. C 59, R3005 (1999); J. Dukelsky and S. Pittel, Phys. Rev. C 63 R061303 (2001); J. Dukelsky, S. Pittel, S. S. Dimitrova and M. V. Stoitsov, Phys. Rev. C 65, 054319 (2002)

[249] T. Papenbrock and D. J. Dean, J. Phys. G 31, S1377 (2005)

[250] J. Rotureau et al., preprint, cond-mat/0603021

[251] M. A. Martín-Delgado and G. Sierra, Phys. Rev. Lett. 83, 1514 (1999)

[252] S. Glazek and K. Wilson, Phys. Rev. D 48, 5863 (1993); 49, 4214 (1994)

[253] T. Eller, H.-C. Pauli and S. Brodsky, Phys. Rev. D 35, 1493 (1987)

[254] G. Fano, F. Ortolani and L. Ziosi, J. Chem. Phys. 108, 9246 (1998), cond-mat/9803071); R. Bursill and W. Barford, Phys. Rev. Lett. 82, 1514 (1999); ibid. preprint, cond-mat/0512649 E. Moore, W. Barford and R. Bursill, Phys. Rev. B 71, 115107 (2005); A. Race, W. Barford and R. J. Bursill, Phys. Rev. B 67, 245202 (2003)

[255] For a review see S. Ramasesha, S.K. Pati, Z. Shuai and J. L. Br'edas, Adv. Quantum Chem. $38,121(2000)$

[256] M. Exler and J. Schnack, Phys. Rev. B 67, 094440 (2003)

[257] B. Normand, X. Wang, X. Zotos and D. Loss, Phys. Rev. B 63, 184409 (2001)

[258] C. Timm and U. Schollwöck, Phys. Rev. B 71, 224414 (2005)

[259] C. Raghu, Y. Anusooya Pati and S. Ramasesha, Journal-ref: J. Phys. A 34, 11215 (2001)

[260] W. Barford and R. Bursill, Chem. Phys. Lett. 268, 535(1997); W. Barford, R. Bursill and M. Lavrentiev, J. Phys: Cond. Matt, 10, 6429 (1998); W. Barford in Ref. 2], Chap.2.3 (Part II) and references therein; M. Lavrentiev, W. Barford, S. Martin, H. Daly, R. Bursill, Physical Review B 59, 9987 (1999); R Bursill and W. Barford, Phys. Rev. B 66, 205112 (2002)

[261] E. Carlon, A. Drzewinski and J. van Leeuwen, J. Chem. Phys. 117, 2425 (2002); Phys. Rev. E 64, 010801(R) (2001); M. Paessens and G. Schütz, Phys. Rev. E 66, 021806 (2002);

[262] M. A. Martín-Delgado, J. Rodriguez-Laguna and G. Sierra, Phys. Rev. B 65, 155116 (2002)

[263] S. White and R. Martin, J. Chem. Phys. 110, 4127 (1999)

[264] S. White in Ref. [2], Chap. 2.1.

[265] Ö. Legeza, J. Röder and B.A. Hess, Phys. Rev. B 67, 125114 (2003); ibid., Mol. Phys. 101, $2019(2003)$ 
[266] S. Daul, I. Ciofini, C. Daul and S. R. White, Int. J. Quantum Chem. 79, 331 (2000)

[267] C. Bauschlicher and P. Taylor, J. Chem. Phys. 85, 2779 (1986)

[268] A. O. Mitrushenkov et al, J. Chem Phys. 115, 6815 (2001)

[269] G. K-L. Chan and M. Head-Gordon, J. Chem Phys. 116, 4462 (2002)

[270] S. Liang and H. Pang, Europhys. Lett. 32, 173 (1995); ibid. Phys. Rev. B 49, 9214 (1994)

[271] S. White and D. Scalapino, Phys. Rev. B 55, 14701 (1997)

[272] S. White and D. Scalapino, Phys. Rev. B 55, 6504 (1997)

[273] S. White and D. Scalapino, Phys. Rev. B 57, 3031 (1998)

[274] E. Arrigoni, A. P. Harju, W. Hanke, B. Brendel and S.A. Kivelson, Phys. Rev. B 65, 134503 (2002); J. Bonca, J. E. Gubernatis, M. Guerrero, E. Jeckelmann and S. R. White, Phys. Rev. B 61, 3251 (2000); A. L. Chernyshev, S. White and A. H. Castro Neto, Phys. Rev. B, 65, 214527 (2002); S. White and D. Scalapino, Phys. Rev. B 61, 6320 (2000); ibid Phys. Rev. B 70, 220506 (2004)

[275] S. White and D. Scalapino, Phys. Rev. Lett. 80, 1272 (1998); ibid. Phys. Rev. B 61, 6320 (2000)

[276] I. P. McCulloch, A. R. Bishop and M. Gulacsi, Phil. Mag. B 81, 1603 (2001)

[277] S. R. White and I. Affleck, Phys. Rev. B 64, 024411 (2001)

[278] M. Q. Weng, et al, preprint, cond-mat/0508186

[279] T. Xiang, J. Lou and Z. Su, Phys. Rev. B 64, 104414 (2001)

[280] N. Maeshima, Y. Hieida, Y. Akutsu, T. Nishino and K. Okunishi, Phys. Rev. E 64 (2001) 016705

[281] M.A. Martin-Delgado, J. Rodriguez-Laguna and G. Sierra, Nucl. Phys. B 601, 569 (2001)

[282] P. Henelius, Phys. Rev. B 60, 9561 (1999)

[283] D. J. J. Farnell, Phys. Rev. B 68, 134419 (2003)

[284] S. Moukouri and L. G. Caron, Phys. Rev. B 67, 092405 (2003); J. V. Alvarez and S. Moukouri, Int. J. Mod. Phys. C 16, 843 (2005); S. Moukouri, J. Stat. Mech. P02002 (2006); S. Moukouri, Phys. Rev. B 70, 014403 (2004)

[285] N. Shibata, J. Phys. A, 36381 (2003)

[286] F. Verstraete et al, preprint, cond-mat/0504305

[287] U. Schollwöck, J. Phys. Soc. Jpn. 74 (Suppl), 246 (2005); see also S. Manmana, A. Muramatsu and R. Noack, AIP Conf. Proc. 789, 269 (2005) (cond-mat/0502396)

[288] M. A. Cazalilla and J. B. Marston, Phys. Rev. Lett. 88, 256403 (2002)

[289] W. H. Press et al, Numerical Recipes in C++, Cambridge Univ. Press, 1993, second edition.

[290] A. Daley, C. Kollath, U. Schollöck and G. Vidal, J. Stat. Mech.: Theor. Exp. P04005 (2004)

[291] H. G. Luo, T. Xiang and X. Q. Wang, Phys. Rev. Lett. 91, 049701 (2003)(comment to 288]); M. A. Cazalilla and J. B. Marston, Phys. Rev. Lett. 91, 049702 (2003) (response to previous comment)

[292] P. Schmitteckert, Phys. Rev. B 70, 121302 (2004)

[293] G. Schneider and P. Schmitteckert, preprint, cond-mat/0601389

[294] A. Feiguin and S. White, Phys. Rev. B 72, 020404 (2005)

[295] S. White and A. Feiguin, Phys. Rev. Lett. 93, 076401 (2004)

[296] G. Vidal, Phys. Rev. Lett. 91, 147902 (2003); G. Vidal, Phys. Rev. Lett. 93, 040502 (2004)

[297] D. Gobert et al, Phys. Rev. E 71036102 (2005)

[298] C. Kollath et al, Phys. Rev. A 71, 053606 (2005)

[299] S. R. Clark and D. Jaksch, Physical Review A,70, 043612 (2004)

[300] C. Kollath, U. Schollwöck and W. Zwerger, Phys. Rev. Lett. 95, 176401 (2005)

[301] E. A. Jagla, K. Hallberg and C. A. Balseiro, Phys. Rev. B 47, 5849 (1993)

[302] T. Oka and H. Aoki, Phys. Rev. Lett. 95, 137601 (2005)

[303] K.A. Al-Hassanieh et al, preprint cond-mat/0601411

[304] A. Micheli, a. j. Daley, D. Jaksch and P. Zoller, Phys. Rev. Lett. 93, 140408 (2004)

[305] T. Kühner and S. White, Phys. Rev. B 60, 335(1999)

[306] Y. Anusooya, S. Pati and S. Ramasesha, J. Chem. Phys. 106, 1 (1997); S. Ramasesha, K. Tandon, Y. Anusooya and S. Pati, Proc. of SPIE, 3145, 282 (1997); S. Ramasesha, Z. Shuai and J. Brédas, Chem. Phys. Lett. 245, 224 (1995)

[307] E. Jeckelmann, Phys. Rev. B 66, 045114 (2002)

[308] E. R. Gagliano and C. A. Balseiro, Phys. Rev. Lett. 59, 2999 (1987).

[309] G. Grosso and G. Partori Parravicini, in Memory Function Approaches to Stochastic Problems in Condensed Matter, Adv. in Chemical Physics, 62, 133 (Wiley, N. Y., 1985)

[310] P. Horsch and W. von der Linden, Z. Phys. B 72181 (1981)

[311] Y. Nishiyama, Eur. J. Phys. B 12, 547 (1999) 
[312] C. L. Zhang, E. Jeckelmann and S. White, Phys. Rev. B 60, 14092 (1999)

[313] W. Q. Yu and S. Haas, Phys. Rev. B, 63, 024423 (2000)

[314] K. Okunishi, Y. Akutsu, N. Akutsu and T. Yamamoto, Phys. Rev. B 64, 104432 (2001)

[315] S. S. Kancharla and C. J. Bolech, Phys. Rev. B 64, 085119 (2001)

[316] H. Matsueda et al, Phys. Rev. B 72, 075136 (2005)

[317] T. Hand, J. Kroha and H. Monien, preprint, cond-mat/0602352

[318] F. Essler, F. Gebhard and E. Jeckelmann, Phys. Rev. B 64, 125119 (2001); E. Jeckelmann, F. Gebhard and F. Essler, Phys. Rev. Lett. 85, 3910 (2000)

[319] E. Jeckelmann, Phys. Rev. B 67, 075106 (2003)

[320] Y.-J Kim et al, Phys. Rev. Lett. 92, 137402 (2004); J. Rissler, F. Gebhard and E. Jeckelmann, J. Phys.: Cond. Matt. 17, 4093 (2005)

[321] H. Benthien, F. Gebhard and E. Jeckelmann, Phys. Rev. Lett. 92, 256401 (2004)

[322] S. Nishimoto and E. Jeckelmann, J. Phys.: Cond. Matt. 16, 613 (2004)

[323] S. Nishimoto, T. Pruschke and R. Noack, J. Phys.: Cond. Matt 18981 (2006)

[324] S. K. Pati and R. Singh, Phys. Rev. B 60, 7695 (1999)

[325] T. D. Kühner, S. White and H. Monien, Phys. Rev. B 61, 12474 (2000)

[326] C. Raas, G. Uhrig and F. B. Anders, Phys. Rev. B 69, 041102(R) (2004); C. Raas and G. Uhrig, Eur. J. Phys. B45(3), 293 (2005)

[327] D. Bohr, P. Schmitteckert and P. Wölfle, Europhys. Lett. 73, 246 (2006)

[328] W. Hofstetter, Phys. Rev. Lett. 85, 1508 (2000)

[329] T. Nishino, J. Phys. Soc. Jpn. 64, 3598 (1995); see also T. Nishino in Ref. 2], Chap. 5(I); T. Nishino and K. Okunishi in Strongly Correlated Magnetic and Superconducting Systems, Ed. G. Sierra and M. A. Martín-Delgado (Springer, Berlin, 1997)

[330] H. Trotter, Proc. Am. Math. Soc. 10, 545 (1959); M. Suzuki, Prog. Theor. Phys. 56, 1454 (1976); R. Feynman and A. Hibbs Quantum Mechanics and Path Integrals (McGraw-Hill, 1965)

[331] R. Bursill, T. Xiang and G. Gehring, J. Phys. Cond. Mat. 8, L583 (1996); X. Q. Wang and T. Xiang, Phys. Rev. B 56, 5061 (1997); N. Shibata, J. Phys. Soc. Jpn 662221 (1997)

[332] A. Gendiar and A. Surda, Phys. Rev. B 63, 014401 (2001)

[333] T. Nishino and N. Shibata, J. Phys. Soc. Jpn. 68, 3501 (1999); T. Enss and U. Schollwöck, J. Phys. A 34, 7769 (2001)

[334] A. Kemper, A. Schadschneider and J. Zittartz, J. Phys. A: Math. Gen. 34, L279 (2001); T. Enss and U. Schollwöck, J Phys A: Math. Gen. 34, 7769 (2001)

[335] R. Baxter, J. Math. Phys. 9, 650 (1968); J. Stat Phys. 19, 461 (1978)

[336] T. Nishino and K. Okunishi, J. Phys. Soc. Jpn. 65,891 (1996); T. Nishino and K. Okunishi, J. Phys. Soc. Jpn. 66, 3040 (1997); T. Nishino, K. Okunishi and M. Kikuchi, Phys. Lett. A 213, 69 (1996)

[337] H. Takasaki, T. Nishino and Y. Hieida, J. Phys. Soc. Jpn. 70, 1429 (2001)

[338] T. Nishino, Y. Hieida, K. Okunishi, N. Maeshima, Y. Akutsu and A. Gendiar, Prog. Theor. Phys. 105, 409 (2001)

[339] C. Ritter and G. von Gehlen, in "Quantization, Gauge Theory and Strings", ed. A.Semikhatov et al., Vol. I, p.563-578, Scientific World Pub. Co. (2001) (cond-mat/0009255

[340] K. Ueda et al, J. Phys. Soc. Jpn. Suppl. 74111 (2005); ibid. J. Phys. Soc. Jpn. 741871 (2005); K. Ueda et al, J. Phys. Soc. Jpn. 75014003 (2006)

[341] N. Shibata and K. Ueda, J. Phys. Soc. Jpn. Vol.70, 3690 (2001)

[342] T. Nishino and K. Okunishi, J. Phys. Soc. Jpn. 67, 3066 (1998); T. Nishino, K. Okunishi, Y. Hieida, N. Maeshima and Y. Akutsu, Nucl. Phys. B 575, 504 (2000)

[343] K. Okunishi, J. Phys. Soc. Jpn. 74, 3186 (2005)

[344] E. Carlon and A. Drzewiński, Phys. Rev. Lett. 79, 1591 (1997); E. Carlon and A. Drzewiński, Phys. Rev. E 57, 2626 (1998); E. Carlon, A. Drzewiński and J. Rogiers, Phys. Rev. B 58, 5070 (1998); A. Drzewiński, A. Ciach and A. Maciolek, Eur. Phys. J. B 5, 825 (1998); A. Maciolek, A. Ciach and A. Drzewiński, Phys. Rev. E 60, 2887 (1999)

[345] A. Drzewiński, A. Maciolek and R. Evans, Phys. Rev. Lett. 85, 3079 (2000); A. Drzewiński, Phys. Rev. E 62, 4378 (2000)

[346] M.-C. Chung, M. Kaulke, I. Peschel, M. Pleimling and W. Selke, Eur. Phys. J. B 18, 655 (2000)

[347] E. Carlon and F. Iglói, Phys. Rev. B 57, 7877 (1998); F. Iglói and E. Carlon, Phys. Rev. B 59, 3783 (1999); E. Carlon, C. Chatelain and B. Berche, Phys. Rev. B 60, 12974 (1999)

[348] J. Kondev and J. Marston, Nucl. Phys. B 497, 639 (1997); T. Senthil, B. Marston and M. Fisher, Phys. Rev. B 60, 4245 (1999); J. Marston and S. Tsai, Phys. Rev. Lett. 82, 4906 (1999); S. Tsai and J. Marston, Ann. Phys. (Leipzig) 8, Special Issue, 261(1999)

[349] M. Kaulke and I. Peschel, Eur. Phys. J. B 5, 727 (1998) 
[350] Y. Hieida, J. Phys. Soc. Jpn. 67, 369 (1998)

[351] E. Carlon, M. Henkel and U. Schollwöck, Eur. Phys. J. B 12, 99(1999); E. Carlon, M. Henkel and U. Schollwöck, Phys. Rev. E 63, 036101 (2001)

[352] J. Hooyberghs, E. Carlon and C. Vanderzande, Phys. Rev. E 62, 036124 (2001)

[353] S.G. Chung, Phys. Rev. B 60, 11761(1999)

[354] Y. Nishiyama, Phys. Rev. E,72, 036104 (2005); ibid. Phys. Rev. E 66, 061907 (2002) and Phys. Rev. E 68, 31901 (2003)

[355] S. Moukouri and L. Caron, Phys. Rev. Lett. 77, 4640 (1996).

[356] S. Moukouri and L. Caron, see Ref. 2, Chap. 4.5(II)

[357] S. Moukouri, preprint, cond-mat/0011169

[358] W. Zhang, J. Igarashi and P. Fulde, J. Phys. Soc. Jpn. 66, 1912 (1997)

[359] R. Bursill, T. Xiang and G. Gehring, J. Phys. C 8, L583 (1996)

[360] H. Trotter, Proc. Am. Math. Soc. 10, 545 (1959); M. Suzuki, Prog. Theor. Phys. 56, 1454 (1976)

[361] X. Wang and T. Xiang, Phys. Rev. B 56, 5061 (1997)

[362] N. Shibata, J. Phys. Soc. Jpn. 66, 2221 (1997)

[363] T. Xiang, Phys. Rev. B 58, 9142 (1998)

[364] N. Shibata, B. Ammon, T. Troyer, M. Sigrist and K. Ueda, J. Phys. Soc. Jpn. 67, 1086 (1998)

[365] K. Maisinger, U. Schollwöck, S. Brehmer, H-J. Mikeska and S. Yamamoto, Phys. Rev. B 58, R5908 (1998)

[366] S. Rommer and S. Eggert, Phys. Rev. B 59, 6301 (1999)

[367] I. Maruyama, N. Shibata and K. Ueda, Phys. Rev. B 65, 174421 (2002)

[368] K. Maisinger and U. Schollwöck, Phys. Rev. Lett. 81, 445 (1999)

[369] B. Ammon, M. Troyer, T. Rice and N. Shibata, Phys. Rev. Lett. 82, 3855 (1999); N. Shibata and H. Tsunetsugu, J. Phys. Soc. Jpn. 68, 3138 (1999)

[370] F. Naef and X. Wang, Phys. Rev. Lett. 84, 1320 (2000)

[371] A. Klümper, R. Raupach and F. Schönfeld, Phys. Rev. B 59, 3612 (1999)

[372] J. Sirker and A. Klümper, Phys. Rev. B 66, 245102 (2002)

[373] Y. Ohta, T. Nakaegawa and S. Ejima, Phys. Rev. B 73, 045101 (2006)

[374] F. Verstraete, J. J. García Ripoll and J. I. Cirac, Phys. Rev. Lett. 93, 207204 (2004)

[375] M. Zwolak and G. Vidal, Phys. Rev. Lett. 93, 207205 (2004)

[376] A. Feiguin and S. White, Phys. Rev. B 72, 220401 (2005)

[377] X. Wang, K. Hallberg and F. Naef in Ref. 2], Chap.7(I)

[378] T. Mutou, N. Shibata and K. Ueda, Phys. Rev. Lett 81, 4939 (1998) (erratum: Phys. Rev. Lett. 82, 3727 (1999))

[379] F. Naef, X. Wang, X. Zotos and W. von der Linden, Phys. Rev. B 60, 359 (1999)

[380] J. Sirker and A. Klümper, Phys. Rev. B 71, 241101 (2005)

[381] G. Kotliar and D. Vollhardt, Physics Today 57, 53 (2004)

[382] J.E. Hirsch and R.M. Fye, Phys. Rev. Lett. 56, 2521 (1986)

[383] M.J. Rozenberg, Phys. Rev. B 55, R4855 (1997)

[384] R. Bulla, Phys. Rev. Lett. 83, 136 (1999)

[385] R. Bulla, T. Costi and D. Vollhardt, Phys. Rev. B 64, 045103 (2001)

[386] D. García, K. Hallberg and M. Rozenberg, Phys. Rev. Lett. 93, 246403 (2004)

[387] A. Georges, G. Kotliar, W. Krauth and M. J. Rozenberg Rev. Mod. Phys. 68, 13 (1996)

[388] Q. Si, M. J. Rozenberg, G. Kotliar, and A. E. Ruckenstein Phys. Rev. Lett. 72, 2761 (1994)

[389] M. J. Rozenberg, G. Moeller and G. Kotliar, Mod. Phys. Lett. B 8, 535 (1994)

[390] See F. Gebhard, E. Jeckelmann, S. Mahlert, S. Nishimoto and R. Noack, Eur. Phys. J B 36 491 (2003), for a previous attempt using a particular algorithm of the DMRG restricted to the small U metallic regime.

[391] S. Nishimoto, F. Gebhard and E. Jeckelmann, J. Phys.: Cond. Matt. 16, 7063, (2004)

[392] A. Georges and G. Kotliar, Phys. Rev. B 45, 6479 (1992)

[393] X. Y. Zhang, M. J. Rozenberg and G. Kotliar, Phys. Rev. Lett. 70, 1666 (1993), A. Georges and W. Krauth Phys. Rev. B 48, 7167-7182 (1993).

[394] D. Garcia, E. Miranda, K. Hallberg and M. Rozenberg, preprint, cond-mat/0608248

[395] F. Alet et al, preprint cond-mat/0410407 http://alps.comp-phys.org/ 